\title{
TTR
}

Traduction, terminologie, re?daction

\section{La déshérence du clandestin : les rites de l'interprétation autour de l'essai sur la traduction de Walter Benjamin}

\section{Laurent Lamy}

Volume 10, numéro 2, 2e semestre 1997

L'essai sur la traduction de Walter Benjamin : traductions critiques Walter Benjamin's Essay on Translation: Critical Translations

URI : https://id.erudit.org/iderudit/037301ar

DOI : https://doi.org/10.7202/037301ar

Aller au sommaire du numéro

Éditeur(s)

Association canadienne de traductologie

ISSN

0835-8443 (imprimé)

1708-2188 (numérique)

Découvrir la revue

Citer cet article

Lamy, L. (1997). La déshérence du clandestin : les rites de l'interprétation autour de l'essai sur la traduction de Walter Benjamin. TTR, 10(2), 87-150. https://doi.org/10.7202/037301ar

\section{Résumé de l'article}

La déshérence du clandestin : les rites de l'interprétation autour de l'essai sur la traduction de Walter Benjamin - À partir d'un corpus restreint, bien délimité, d'analyses critiques consacrées à l'essai sur la traduction de Benjamin, cette étude dégage une diversité de points de vue qui, sous leur contradiction apparente, en appellent de la part du lecteur à un processus de traduction continue qui est pour ainsi dire encodée, de façon intrinsèque, dans la poétique même de Benjamin. Loin de se vouloir un cas d'espèce enveloppé dans une aura ésotérique, cette façon de résister à toute forme d5explaining away, indique plutôt que toute théorie de la traduction doit elle-même se « traduire » dans l'élément où elle tente de s'expliquer.
Tous droits réservés (C) TTR: traduction, terminologie, rédaction — Les auteurs, 1997
Ce document est protége par la loi sur le droit d'auteur. L'utilisation des services d'Érudit (y compris la reproduction) est assujettie à sa politique d'utilisation que vous pouvez consulter en ligne. 


\section{La déshérence du clandestin : les rites de l'interprétation autour de l'essai sur la traduction de Walter Benjamin}

\section{Laurent Lamy}

" En tant qu'orientation absolue vers l'Autre - en tant que sens l'œuvre n'est possible que dans la patience, laquelle, poussée à bout, signifie, pour l'Agent : renoncer à être le contemporain de l'aboutissement, agir sans entrer dans la Terre Promise. " Emmanuel Lévinas, Humanisme de l'autre homme

S'il est une cuvre qui s'est imposée, dans sa fragmentation et son inachèvement, par la rigueur et la pénétration de sa faculté critique ainsi que l'intransigeance de son traitement micrologique d'éléments les plus disparates, c'est bien celle de Walter Benjamin. Son tracé converge au foyer d'un prisme dont le spectre découvre des nuances si subtiles et inattendues que leur perception fait encore difficulté pour ceux et celles qui auront appris à fréquenter l'entrelacs de ses arcades, surpris à déchiffrer des arcanes pourtant livrées au grand jour; Benjamin est un passager clandestin de ce siècle, et son œuvre, toujours en état de déshérence. Elle 
commande une attitude plutôt que les fantasmes d'une adhésion qui sera toujours déçue par sa maîtrise impénitente de la contradiction. Il aura, sans doute malgré lui, ébauché une méthode de survie pour une postérité orpheline de tout ascendant tutélaire en matière de culture, de connaissance et d'orientation sociopolitique. Né sous le signe de Saturne et adepte d'un messianisme pleinement sécularisé, il n'aura jamais espéré pour lui-même ne serait-ce qu'une infime parcelle de la Terre promise. Il ne ressemble à personne et c'est pourquoi nous luj ressemblons.

Dans son introduction à la toute première édition des Schriften de Benjamin, parus en 1955, Theodor Adorno met en relief deux considérations essentielles à l'approche de sa pensée ${ }^{l}$. D'abord, sa relation intensive au concret, que jamais il n'abandonne à la gouverne du concept, lui reconnaissant plutôt cette densité expressive qui, dans sa teneur originelle, ne saurait se laisser inféoder à une forme quelconque de visée intentionnelle la subordonnant à l'activité cognitive d'un sujet.

Un second point de tension concerne la matière vive de son œuvre qui, de son propre aveu, s'est toujours voulue de nature littéraire et historique. Comme le souligne Adorno, la méfiance de Benjamin à l'endroit de la métaphysique, notamment dans sa tangente jdéaliste, l'amènera à prohiber toute approche de l'absolu qui fasse l'économie de l'histoire, laquelle ne saurait être désormais conçue à l'instar d'une odyssée hégélienne.

Il s'agit, à l'évidence, d'une pensée qui est à l'essai et qui

' Theodor W. Adomo, "Einleitung zu Benjamin's Schriften ", in W. Benjamin, Schriften, éd par T. W. Adomo et Gretel Adorno en collaboration avec Friedrich Podszus, Frankfurt am Main, Suhrkamp, 1955, pp. 9-27; trad. anglaise par R. Hullot-Kentor, in Gary Smith (dir.), On Walter Benjamin. Critical Essays and Recollections, Cambridge, Mass., MIT Press, 1988, pp. 2-17; nous nous référons aux pp. 7-9 de la traduction anglaise. 
excellera dans le genre présumément hybride de l'essai. Mais les " lois " du genre empruntent ici des avenues insoupçonnées, où l'on poursuit les linéaments tremblants et troubles d'une fresque dont les couches successives nous découvrent un véritable laboratoire de la modernité - une modernité déjà lourde des présages qui allaient voir des millions de visages s'engouffrer dans les camps de la mort, et ce sous le silence complice, dans la sourde torpeur d'une Europe usée et rompue aux nihilismes de tout acabit. Parmi les dispositifs critiques mis en cuvre dans cet atelier où, sans abuser, nous pourrions rencontrer l'ange désabusé de la Melencolia de Dürer, on trouve l'essai plutôt controversé que Benjamin consacrait en 1921 à Die Aufgabe des Öbersetzers. Son sujet est le sujet même de la traduction, qui se voit ici purgée de toute instanciation subjective au profit de la vie immanente des œuvres. Son enjeu est l'appel qui commande celui ou celle qui est mandaté par cet enjeu qui, paradoxalement, n'est l'apanage ni de la source qui sollicite ni de l'atteinte du résultat qu'on en escompterait. C'est là la difficulté, notre difficulté. Et sa mesure serait celle d'un abandon.

La réception d'une ouvre trahit souvent davantage les auspices sous lesquels s'est engagée cette réception, les attentes de l'instance réceptrice, que ce qui fait l'objet de cette réception. En revanche, il est des ouvres qui trompent ces attentes et les acculent même à leurs derniers retranchements. L'essai sur la traduction de Benjamin est tout à fait exemplaire à cet égard. C'est un morceau de littérature qui tend à éconduire, c'est-à-dire que sa réception condamne à la déception, et ce de la première à la dernière ligne. In limine, il est affirmé que la réception d'une cuvre ne lui est pas essentielle, tout au plus anecdotique; davantage encore, la considération du facteur humain pour ce qui est de la réalité et de l'intelligence même de l'œuvre est sinon superflue, à tout le moins relative à un présupposé que l'on peut facilement répudier. In fine, notre homme soutient que la version interlinéaire des Écritures ou d'un texte sacré est l'archétype ou l'idéal de toute traduction, mettant ainsi en faillite, du moins le semble-t-il à la première 
lecture, toute velléité de création en regard d'une matrice qui comporterait en elle-même son propre magistère. De Charybde en Scylla, ou la quadrature du cercle.

Benjamin fausse ainsi compagnie à deux courants de pensée dominants en matière d'interprétation et d'exégèse. D'une part, les tenants d'une " esthétique de la réception * (Rezeptionsästhetik), mise de l'avant notamment dans les travaux de Hans Robert Jauss et de Wolgang Iser ${ }^{2}$ et, de l'autre, la perspective historico-critique de l'herméneutique contemporaine, qui s'inscrit dans le sillage des travaux de Hans-Georg Gadamer ${ }^{3}$. L'étonnement n'est pas moindre lorsqu'on se laisse entraîner dans l'entrechoc des thèses qui jalonnent ce texte. Benjamin affirme entre autres que la traduction est le lieu privilégié d'une régénération inespérée des œuvres, donc qu'elle se veut garante d'une vie qui leur échoit de surcroit, et qu'elle participe ainsi à la " croissance sacrée " des langues vers le terme messianique de leur histoire, s'agissant alors d'éveiller en elles, dans le contact avec l'étranger, les germes du " pur langage ", bref de libérer les harmoniques de la reine Sprache qui s'y trouve en quelque sorte disséminée. Il est un autre point auquel Benjamin accorde un statut proprement canonique, à savoir que l'ordre du vivant, tout ce à quoi on reconnaît une vie, ne saurait être conçu en faisant abstraction de l'histoire; de façon plus précise, c'est à partir de l'histoire que la sphère de la vie reçoit sa pleine et entière

${ }^{2}$ Voir Hans Robert Jauss, Pour une esthétique de la réception, trad. par Cl. Maillard, Paris, Gallimard, 1978; Wolfgang Iser, I'Acte de lecture. Théorie de l'effet esthétique, trad. par E. Sznycer, Bruxelles, Pierre Mardaga éditeur, 1985.

${ }^{3}$ Notons la publication récente de la traduction intégrale, par les soins de Pierre Fruchon, Jean Grondin et Gilbert Merlio, du maître-ouvrage de Gadamer, Vérité et méthode. Les grandes lignes d'une herméneutique philosophique (Paris, Seuil, 1996); en même temps paraissaient, dans une édition établie par P. Fruchon, les conférences prononcées en français en 1958 par Gadamer, sous le titre : le Problème de la conscience historique (Paris, Seuil, 1996). 
détermination. Le lecteur doit vivre avec le paradoxe voulant que le texte sacré jouisse d'une immunité absolue face à toute modalité d'interprétation, au premier chef celle de la traduction, alors que, d'un autre côté, il n'est de signification qui fasse sens et connaisse une vie propre sans se ressourcer à la dynamique de l'histoire qui, dans ce cas, loin seulement de cautionner le relativisme s'attachant à la prévalence de tel ou tel autre paradigme, n'est investie de sa réelle dimension que dans la perspective d'une eschatologie messianique dont l'horizon demeure ouvert à l'infini. Et il va sans dire que l'idée de progrès constituait pour Benjamin une chimère bien avant qu'elle ne soit broyée dans les rouages de l'univers concentrationnaire. Ce sont là les lignes de force d'une argumentation à caractère transcendantal qui trouve son assise dans l'autonomie absolue d'une vie langagière qui, pour autant que les cuvres y donnent accès, doit être dessaisie de tout influx d'ordre subjectif si l'on entend percer à jour l'impératif secret qui commande l'exercice de la traduction.

Dans ses Études sur la philosophie de Walter Benjamin ${ }^{4}$, Rolf Tiedemann, l'éditeur avec Hermann Schweppenhäuser des Gesammelte Schriften de Benjamin, aborde la problématique du langage chez Benjamin et le rôle crucial qu'y jouera la théorie de la traduction à partir de sa critique de l'idéalisme kantien. Benjamin y déplore un appauvrissement marqué d'une dimension qui sera pour lui toujours irréductible, celle de l'expérience. Il est particulièrement allergique à la a mythologie * du sujet connaissant, substantiel ${ }^{5}$, qui hante toujours le criticisme kantien.

${ }^{4}$ Rolf Tiedemann, Études sur la philosophie de Walter Benjamin, preface de T. W. Adorno, trad. par Rainer Rochlitz, Arles, Actes Sud, 1987.

${ }^{5}$ Adorno rappelle en effet que, dès le début, la pensée de Benjamin * se révolte contre le mensonge selon lequel l'homme et l'esprit humain auraient leur fondement en eux-mêrnes et produiraient un absolu. (...) Au cours d'un entretien, Benjamin n'accorda de valeur qu'au soi mystique, non au soi métaphysique et gnoséologique, "substantiel" " (Prismes, trad. par G. et R. Rochlitz, Paris, Payot, 
De la même façon, l'idée ne sera plus pour Benjamin un principe régulateur au service d'un sujet appelé à constituer le champ phénoménal sur la base de l'unité synthétique de l'aperception transcendantale; l'idée est immanente à l'être même de l'étant, c'est elle qui permet le a sauvetage * (Rettung) des phénomènes dans leur singularité aussi bien que dans leur pluralité qui, loin de se laisser réduire à l'unité fictive du jugement, en appelle à l'harmonie profonde de la * langue des noms * (Namensprache) comme refuge de la face symbolique cachée, de la prégnance du symbole dans les formes de la vie langagière, et comme foyer intensif de toute signification. Le nom concentre en lui toutes les virtualités de la traduction. Comme l'écrit Benjamin dans la Vorrede de son étude sur l'Origine du drame baroque allemand, la structure de la vérité exige "un être qui égale par son absence d'intentionnalité l'être simple des choses ", et cet être est le nom ${ }^{6}$.

Le nominalisme singulier de Benjamin est étranger à toute théorie conventionnaliste de la signification. Les idées ne sont pas des créatures de l'intelligence destinées à valider dans les phénomènes ce qui échappe à la juridiction du concept et qui, de ce fait, n'auraient d'autre consistance que celle que leur accorde l'usage d'un système arbitraire de signes. En effet, écrit Tiedemann, Benjamin " localise l'Idée à un autre niveau du langage : là où il n'impose pas de significations aux phénomènes, au nom d'un arbitraire subjectif, mais où il appartient "au cour même de la réalité", qu'il s'agit de "percevoir" " (1987, p. 44); davantage encore, " c'est en tant qu'élément langagier seulement que quelque chose comme l'être, ou plus précisément : un mode de l'être, est donné * (Ibid., p. 47). En ce sens, comme le suggérait son ami Gershom Scholem dans une lettre qu'il lui adressait de Jéricho

1986, p. 207).

${ }^{6} \mathrm{~W}$. Benjamin, Origine du drame baroque allemand, trad. par Sibylle Muller (avec le concours d'André Hirt), préface d'Irving Wohlfarth (Paris, Flammarion, 1985), p. 33. 
le 31 mars 1931, Benjamin est bien " l'héritier légitime des traditions les plus fécondes et les plus authentiques d'un Hamann et d'un Humboldt $x^{7}$. Déjà, dans un essai rédigé en 1918 et resté inédit de son vivant, "Über das Programm der kommenden Philosophie ", Benjamin évoquait le débat engagé par Johann Georg Hamann avec Kant ${ }^{\sharp}$, qui met en cause chez ce dernier l'absence de toute problématisation du langage, lequel constitue pour Hamann la matrice de l'univers intelligible et doit par conséquent apporter le correctif nécessaire à une mathématisation unilatérale du monde de la nature dans une architectonique de la raison qui ignore la fécondité inépuisable du Verbe révélé. Par ailleurs, dans des notes de lecture rédigées en 1925, Benjamin relève la thèse de Humboldt selon laquelle il existe une a relation de réciprocité ténue, et jamais pleinement saisissable, entre

${ }^{7}$ W. Benjarnin, Briefe, éd. et annotés par Gershom Scholem et T. W. Adorno (Frankfurt am Main, Suhrkamp, 1966), p. 526.

${ }^{8}$ Benjamin y écrit en effet (" Sur le programme de la philosophie qui vient ", trad. par Maurice de Gandillac, Oeuvres I: Mythe et violence, Paris, Denotl, 1971, p. 111) : \& La grande transformation et la grande rectification à laquelle il convient de soumettre le concept de connaissance orienté de façon unilatérale vers les mathématiques et la mécanique n'est possible que si la connaissance est mise en relation avec le langage, comme Hamann l'avait tenté du vivant même de Kant . Sur l'influence des vues de Hamann sur celles de Benjamin en matière de langage, voir Winfried Menninghaus, Walter Benjamins Theorie der Sprachmagie (Frankfurt am Main, Suhrkamp, 1980), pp. 21-33 et 205-215.

${ }^{9}$ Pour un excellent aperçu de la personnalité et des thèses de Hamann, voir le bel ouvrage d'Isaiah Berlin, The Magus of the North. J. G. Hamann and the Origins of Modern Irrationalism, éd. par Henry Hardy (New York, Farrar, Straus and Giroux, 1994); pour un aperçu plus détaillé des recherches de Hamann sur le langage, voir Volker Hoffman, Johann Georg Hamanns Philologie. Hamanns Philologie zwischen enzyklopädischer Mikrologie und Hermeneutik (Stuttgart, 1972). 
l'expression et la pensée. "10 Or, comme le souligne Rolf Tiedemann, « la compréhension de cette relation réciproque, de la pensée médiatisée par le langage, du contenu du langage pour autant qu'il est médiatisé par la forme du langage, est centrale pour la théorie du langage de Benjamin » (1987, p. 48).

Quoique Tiedemann n'y insiste pas davantage, le détail important ici, c'est la médiatisation du contenu par la forme, qui est un postulat essentiel de la théorie de la traduction élaborée par Benjamin. La traduction doit d'abord s'attacher au " mode de la visée " (die Art des Meinens) qui s'opère dans la langue à traduire plutôt qu'au contenu qui est visé (das Gemeinte) à travers cette langue et qui, suivant une conception encore largement partagée, serait en quelque sorte le tuteur transcendantal en matière de traduction. Pour Benjamin, on ne saurait faire l'économie de la forme au profit du contenu et même l'en dissocier puisque c'est le devenir même du langage, bref - pour y aller d'une précision de notre cru - l'affinement et l'intensification de son coefficient de densité expressive, qui est en jeu dans le travail de la traduction. C'est une leçon que Henri Meschonnic a faite sienne dans sa poétique de la traduction qui, loin de plaider pour un plat littéralisme ${ }^{11}$, exige entre autres que l'on prenne la mesure de la dynamique interne du rythme, de la scansion qui lie le signifiant au signifié, révoquant ainsi toute opportunité de conclure à un quelconque ascendant de l'un sur l'autre. Le rapport intensif qui lie la forme à la signification est le point d'incidence de la densité des ruptures historiques encodées dans les états de la langue, en même temps que le vecteur discret des métamorphoses encourues dans la

\footnotetext{
${ }^{10}$ W. Benjamin, « Reflexionen zu Humboldt „, Gesammelte Schriften, Vol, VI, éd. par Rolf Tiedemann et Hermann Schweppenhauser (Frankfurt am Main, Suhrkamp, 1985), p. 27 : " Humboldt spricht von dem "feinen, und nie völlig zu begreifenden Wechselverhältnis des Ausdrucks und des Gedankens." "

${ }^{11}$ Voir l'importante mise au point de Meschonnic dans * Traduire ce que les mots ne disent pas, mais ce qu'ils font * (Meta, Vol. XL, $n^{\circ} 3,1995$ ), pp. 514-517.
} 
livraison de factures poétiques tributaires du rapport à l'étranger, donc d'une fécondation qui restitue les constructions finies de la langue à la virtualité originelle de leurs ressorts expressifs. La prégnance historique de l'alternative entre mimésis et convention, qui remonte au débat amorcé dans le Cratyle de Platon ${ }^{12}$, se voit ainsi frappée de caducité.

Ce point est crucial pour Benjamin, car il ne saurait être question pour lui de faire du langage une hypostase du réel. Si Benjamin s'oppose à une conception purement sémiotique ou conventionnaliste du langage, il n'est pas davantage réceptif à la conception mystique qui fait du langage une composante ontologique de la réalité. Si le langage participe de l'être des choses, il ne coïncide pas avec elles; il leur est inhérent sur un mode purement virtuel, comme condition d'accès à leur dénomination, comme puissance latente du nom appelé à communiquer leur essence spirituelle.

L'arrière-plan théologique de cette thèse est développé dans un essai rédigé en 1916 et demeuré lui aussi inédit du vivant de Benjamin, "Über Sprache überhaupt und uber die Sprache des Menschen ${ }^{13}$. Il est opportun ici d'ouvrir une parenthèse et d'exposer les grandes lignes de son argumentation ainsi que certains aspects de l'articulation qui lui est sous-jacente, puisque cet essai recèle quelques-unes des clefs essentielles à la compréhension de la théorie de la traduction chez Benjamin. Il y met formellement en question toute approche du fait langagier, de l'être même du langage, qui le réduirait à une fonction ancillaire, à la servilité d'un moyen de communication, d'une simple courroie

\footnotetext{
${ }^{12}$ Voir à ce sujet Gérard Genette, Mimologiques, Voyage en Cratylie, (Paris, Seuil, 1976).

${ }^{13}$ Voir Gesammelte Schriften, II, 1 (éd. par R. Tiedemann et H. Schweppenhäuser, 1977), pp. 140-157; trad. par M. de Gandillac, " Sur le langage en général et sur le langage humain ", Mythe et violence, pp. 79-98.
} 
de transmission. Il correspond plutôt à un médium, à un milieu ou à une matrice où l'essence spirituelle des choses se révèle proportionnellement à la densité expressive du nom qui est appelé à la communiquer. Mais cette thèse emprunte un tour encore plus radical, qui marque l'insuffisance même de la notion d'expression, dans la mesure où cette dernière dénote un mouvement d'extériorisation en direction d'un référent. C'est pourquoi Benjamin s'en remet à un rapport de proportionnalité (Verhältnis der Proportionalität) pour qualifier le lien entre l'essence spirituelle (das geistige Wesen) et le langage dans lequel elle se communique : elle se communique dans le langage et non par lui. L'essence spirituelle n'est pas identique au langage où elle se communique, mais * ce qui est communicable dans une essence spirituelle est son langage " (Mythe et violence, p. 81).

Évoluant aux antipodes de toute théorie du langage dominée par l'arbitraire du signe, Benjamin convient de l'immédiateté originelle de la vie langagière, de son inhérence exclusive en regard de tout contenu extérieur à sa manifestation : « $l$ n'existe rien de tel qu'un contenu du langage; en tant que communication, la langue communique une essence spirituelle, c'est-à-dire purement et simplement une communicabilité * (Ibid., p. 85, trad. modifiée; c'est Benjamin qui souligne).

Si la traductibilité (Übersetzbarkeit) est sans conteste le concept-clef de l'essai sur la traduction, celui de communicabilité (Mitteilbarkeit) est le pivot autour duquel s'organise l'argument transcendantal de l'essai de 1916. Puisqu'il faut constater l'existence d'une pluralité irréductible de langues et d'un nombre indéfini de différences entre leurs divers champs d'expression, leur distinction sera pour Benjamin de l'ordre de la densité (Dichte - on fera le rapprochement ici avec Dichtung); c'est-à-dire que leur degré d'existence respectif et leur distribution dans la hiérarchie de l'être se veulent graduels et proportionnels à la teneur spirituelle qu'elles savent communiquer. La dimension langagière comme pure communicabilité, comme pur médium encore vierge de tout 
contenu visé par une intention signifiante empruntant la forme d'un jugement, n'est autre que l'analogon de la dimension spirituelle. Le recours à l'analogie permet d'échapper à toute conception métaphorique du langage. Autrement dit, le langage n'est pas la représentation d'un " sujet parlant » ou d'un a locuteur du langage » (Sprecher der Sprache), comme l'écrit Benjamin. Le langage ne peut faire l'objet d'une représentation parce qu'il est irreprésentable : ce qui permet la " présentation " (Darstellung) ne se représente pas. La faillite de la représentation à l'endroit même de la langue, du fait qu'il y a des langues et que l'on ne peut se situer en dehors d'elles pour s'en enquérir, signifie que la communicabilité est l'incommunicable même, tout comme la traductibilité se veut elle-même intraduisible et commande comme telle la traduction, lui assigne son lieu, la possibilité qu'elle ait lieu sur la base même de son impossibilité. $\mathrm{Si}$, comme le conçoit Benjamin, chaque idiome affiche un degré de densité variable quant à ses ressources expressives, il appartient alors à la traduction d'actualiser dans l'incommensurabilité même de ces variations le frayage intensif, à peine perceptible, des harmoniques de la reine Sprache, bref de ce que Benjamin désignera dans son essai sur l'Aufgabe du traducteur comme le " pur langage ".

C'est ici que Benjamin fait intervenir le concept de révélation (Offenbarung), dans le cadre d'un commentaire sur les premiers chapitres de la Genèse hébraîque. La parole révélée, en tant qu'elle procède du verbe créateur, est le paramètre absolu de la communicabilité investie dans la " Jangue des noms * (Namensprache). Benjamin insiste en premier lieu sur le rapport insigne qui existe entre le fiat divin, la prodigalité du verbe créateur, et le " don du langage " (die Gabe der Sprache) à l'homme, qui se voit alors habilité à nommer les choses : le prototype adamique n'est pas le produit du verbe divin, mais est inséminé par son souffle, il reçoit ce verbe, il en est le véhicule actif, l'incorporation du dessein divin. Dieu n'a pas donné un nom à l'homme, mais la possibilité de connaître la puissance créatrice du verbe dans le nom, dans l'acte de dénomination (Benennung). 
La trace de cette prodigalité originelle du verbe est encryptée dans le nom propre (der Eigenname), qui marque la communauté de l'homme avec la puissance créatrice du verbe divin. Le nom propre dessine la frontière intérieure entre la créativité infinie de la langue et la finitude de son exercice, celle qui correspond à l'exil de la créature, à son extradition hors du règne marqué par la « langue des noms $n$. L'impossibilité de traduire le nom propre est l'emblème silencieux de la traduction originaire qui commande toutes les traductions, qui les situe toujours à la frontière d'une communicabilité qui permet de communiquer parce qu'elle-même $s^{\prime}$ avère incommunicable ${ }^{14}$.

Le nom s'est révélé créateur comme verbe, nous dit Benjamin, et ce verbe est source de connaissance comme nom. Cette connaissance n'est cependant pas le fruit d'une " génération spontanée "(spontane Schopfung), "elle ne surgit pas du sein de la langue, à l'instar de la création, de manière absolument illimitée et infinie; par contre, le nom que l'homme restitue à la langue dépend de la façon dont ce nom se communique à lui " (Ibid., p. 90; trad. modifiée). Il s'agit véritablement d'une " conception " au sens matriciel ou utérin du terme, et cette gestation de la langue dans le nom est le mandat qui est confié de façon privilégiée à l'exercice de la traduction qui, en l'occurrence, désigne non seulement la translation entre une langue-source et une languecible, mais la " conception dans le nom de ce qui est sans nom * (Empfängnis des Namenlosen im Namen). Or, poursuit Benjamin, " en vertu du rapport entre les langues plus haut caractérisé comme celui de médias affichant une densité variable, on conclut à la traductibilité des langues les unes dans les autres. La traduction est le transfert d'une langue dans une autre à travers un continuum de métamorphoses. La traduction traverse des continuums de

\footnotetext{
${ }^{14}$ Voir à ce sujet Laurent Lamy, * Le don et l'abandon : la trace de la traduction dans la blanche écriture du concept ", Le Mur et l'arcade. Essais sur la traduction, éd. par Alexis Nouss, à paraître.
} 
métamorphose, non pas des zones abstraites d'équivalence et de ressemblance "(Ibid., p. 91; trad. modifiée).

Si l'homme ne peut que donner aux choses le nom que cellesci lui communiquent, c'est qu'en elles précisément a le verbe créateur est le germe du nom connaissant " (das schaffende Wort in ihnen ist der Keim des erkennenden Namens). Bref, la " communication virtuelle de la mutité " (die mitteilende Stummheit) des choses au langage des mots mobilisé par l'homme recèle en elle le principe de la connaissance, qui s'accomplit dans le nom. I1 n'en faut pas plus pour justifier l'existence d'une pluralité de langues et, de là, convenir de la nécessité de la traduction comme médium paradigmatique de la connaissance appelée à réactualiser la puissance créatrice du verbe encrypté dans le nom : " L'amplitude infinie du retard qu'accuse la mutité du verbe dans l'existence des choses par rapport au verbe qui, dans la gnose de l'homme, leur confère un nom, et le retard qu'accuse à son tour ce dernier par rapport au verbe créateur de Dieu, sont au fondement même de la pluralité des langues humaines. C'est uniquement dans la traduction que le langage des choses peut s' introduire dans la langue de la connaissance et du nom - autant de traductions, autant d'idiomes, depuis l'instant même où l'homme s'est vu une fois pour toutes déchu de la condition paradisiaque, laquelle ne connaissait qu'une seule langue " (Ibid., pp. 92-93; trad. modifiée).

Pour Benjamin, il ne s'agit pas simplement de désavouer une conception du langage qui se résout dans la projection du sujet dans l'objet par le biais d'une représentation consignée dans un médium déterminé par un décret arbitraire de l'entendement et qui permettrait ainsi de valider sa perception des phénomènes sous la forme d'un jugement confortant les assises de sa raison. Benjamin s'oppose d'abord à la réification inhérente à la communication entre les hommes pour lui arracher les vestiges d'un monde à jamais perdu. “ Il est déterminant pour la pensée de Benjamin, écrit Rolf Tiedemann, que la sphère du nom soit aussi celle qui, une fois 
pour toutes, est perdue; en d'autres termes, le langage n'est pas élevé au rang de révélation de l'être. Il n'est pas immédiatement identique à l'être des ontologies, et n'est donc pas ce qui "donne", "offre", "fait don"; il ne dispose pas d'une "force d'appellation intacte" dont la pensée disposerait alors à son tour, ce qui lui permettrait de la "reconquérir" " (1987, p. 57). Tiedemann use délibérément ici de formules appartenant au lexique heideggerien, puisqu'il décèle une opposition très nette entre la conception du langage chez Benjamin et celle dont Heidegger s'est fait le héraut après son rectorat de funeste mémoire. Fort d'une interprétation partiale, pour ne pas dire biaisée des poétiques de Hölderlin et de Trakl, Heidegger s'adonne à une hypostase ontologique de certains vocables, de certaines racines étymologiques dont le spectre sémantique s'enveloppe dans l'atmosphère " numineuse * ou oraculaire d'une auto-révélation de l'être celé sous le voile du néant. Évidemment, il eût été le premier à s'en défendre, puisqu'il y va toujours de l'être qui, à l'instar du néant, n'est pas un étant, rien qui soit. Quoi qu'il en soit, Benjamin s'oppose à toute idolâtrie du langage, puisque l'exil du Nom divin hors de la sphère du dicible interdit l'accès à toute dénomination originelle de la vérité révélée.

Mais l'opposition la plus profonde entre la pensée de Benjamin et celle de Heidegger se situe au niveau de l'origine, dans sa conception même. « La "pensée originaire" de Heidegger vise l'archaïque, l'être de l'ontologie comme initium : faire surgir quelque chose d'un bond qui devance, l'amener à être à partir de la provenance essentielle et dans le saut instaurateur, voilà ce que nous signifie le mot origine ». Il vise, selon le terme créé par Benjamin - employé par Heidegger dans ses commentaires sur Hölderlin -, le * dictamen * (das Gedichtete) ou, selon la terminologie de l'interprétation de Trakl, " le Dict * (das Gedicht), par opposition aux poèmes singuliers d'un auteur (1987, pp. 9293). Pas besoin de dire ici que Heidegger joue sur le rapport incestueux entre les vocables Sprung (saut) et Ursprung (origine). La problématisation de l'origine chez Benjamin s'inscrit dans une 
direction diamétralement opposée : à jamais perdue, elle ne saurait coïncider avec l'" intact ", l'" indemne " ou le " sauf ", dont on sait qu'ils comptent parmi les nuances du spectre sémantique que Heidegger assortit à l'expression allemande das Heilige : "le sacré $n$.

" [...] Ce qui importe pour lui, c'est toujours le nouveau, ce en quoi toute cuvre d'art surgit d'un stade antérieur de l'histoire; c'est le contenu de vérité qui est "essentiellement Résultat, c'est-àdire qu'il est à la fin seulement ce qu'il est en vérité" " (1987, p. 93). L'interprétation de Tiedemann est tout à fait juste sauf que, disciple d'Adorno, il se prévaut d'une formule hégélienne pour traduire une idée qui s'inscrit plutôt dans la perspective d'une eschatologie messianique en rupture avec toute finalité immanente de l'histoire, puisque celle-ci ne présente qu'un amas de ruines, un site déserté par la félicité du contact originel, immédiat, avec la munificence du Nom divin. La maxime de Karl Kraus qui sert d'épigraphe à la XIV ${ }^{e}$ Thèse sur la philosophie de l'histoire de Benjamin est nettement plus indiquée : L'origine est la fin ${ }^{15}$. Ainsi la teneur originelle d'une auvre poétique, bref l'intensité de son rapport à l'origine, ne peut-elle se révéler qu'en trouvant asile dans le mouvement d'exode qu'entraîne sa traduction dans une langue qui pour l'accueillir doit connaître les douleurs de l'enfantement dans son propre élément.

De la même façon, le quantum de densité affichée par les ressources expressives d'une œuvre est proportionnel à sa traductibilité qui, à son tour, mesure l'abîme qui sépare la singularité incarnée dans sa poétique de la plénitude à jamais perdue de la langue dans laquelle s'est produite la révélation. Le fait qu'il soit impossible de restituer dans leur intégrité originelle les harmoniques de cette langue, donc que la sphère du nom soit à

$15 \mathrm{~W}$. Benjamin, « Theses sur la philosophie de l'histoire ", trad. par M. de Gandillac, Oeuvres II : Poésie et révolution (Paris, Denoèl, 1971), p. 284. 
jamais perdue, est le moteur de la conception messianique de l'histoire chez Benjamin.

L'écheveau des thèses, par moment assez déconcertantes, qui ponctuent l'essai sur la traduction est tributaire de cette conception, qui n'est évidemment pas étrangère aux nombreuses discussions que Benjamin a eues avec son ami Gershom Scholem sur la théorie kabbalistique du langage. Dans le très beau mémoire consacré à l'histoire de son amitié avec Benjamin, Scholem rapporte qu'au tournant des années 1920-1921, alors qu'il lui faisait part des difficultés rencontrées dans ses recherches sur la théorie mystique du langage dans la Kabbale, en particulier dans son étude des ouvrages d'Abraham Aboulafia ${ }^{16}$, Benjamin montra à son grand étonnement le plus vif intérêt pour ces recherches ${ }^{17}$. Lui-même se préoccupait intensément de philosophie du langage à cette époque, puisqu'il comptait alors rédiger une thèse sur la théorie de la connaissance. Les questions soulevées lors de ces

\footnotetext{
${ }^{16}$ Abraham Aboulafia (1240-c. 1291) est un mystique juif originaire d'Espagne, promoteur d'une variété prophétique de la Kabbale basée sur l'apprentissage de la concentration (hitbodedut) et de techniques respiratoires, la pratique de la musique et, de façon toute particulière, l'approfondissement de la combinatoire des noms divins qu'il associe à l' $\propto$ œuvre du Chariot " (ma'aseh merkovah), soit l'ascension de l'âme (il s'agit en réalité d'une descente à l'intérieur de soi) vers le royaume visionnaire contemplé par le prophète Ezéchiel. Frappé d'hérésie, Aboulafia fut très tôt banni du cercle des kabbalistes orthodoxes d'Espagne. Mais son auvre s'est disséminée jusqu'au Proche-Orient, à travers les cercles kabbalistiques italiens et byzantins, pour connaître une heureuse fécondation au contact de la mystique soufie (Islam) et du néoplatonisme alexandrin, et enfin rayonner jusqu'en terre d'Israêl, au XVI' siècle, dans l'école de Safed, où elle demeure une source d'inspiration pour les auvres non moins originales de Moïse Cordovéro (1522-1570) et d'Isaac Louria (1534-1572). Voir à ce sujet les travaux de Moshe Idel, The Mystical Experience in Abraham Abulafia (Albany, State University of New York Press, 1987); Language, Torah, and Hermeneutics in Abraham Abulafia (Albany, SUNY Press, 1988).
}

${ }^{17}$ G. Scholem, Walter Benjamin. Histoire d'une amitié, trad. par Paul Kessler, notes de Roger Errera (Paris, Presses Pocket, Coll. « Agora » [Calmann-Lévy] 1989), p. 43. 
discussions avec Scholem ne pouvaient manquer d'affecter la rédaction, la mềme année, de ses prolégomènes à la traduction de Baudelaire, et viendront par la suite alimenter le filon qui s'est cristallisé dans la "Préface épistémo-critique » de l'étude sur le Trauerspiel, parue en 1928.

Il ne fait aucun doute que la spéculation kabbalistique sur l'origine du langage, selon laquelle notamment cette faculté dévolue à l'homme et susceptible d'être pleinement épanouie en lui en tant que symbole vivant du royaume créaturial est tout entière concentrée et anticipée en Dieu, dans la contemplation du Nom divin, est une composante essentielle de la conception du langage chez Benjamin. Selon Scholem, un des postulats de cette spéculation est la conviction qu'il existe un élément proprement incommunicable, un aspect interne du langage qui n'atteint pas le stade de l'expression dans le rapport entre les êtres. C'est-à-dire que la couche sémiotique de l'expression, sa valeur de communication et son intégration dans un ordre de la signification ne suffisent pas à épuiser les ressorts de la vie langagière; davantage encore, cette dimension inexprimée, cette part insaisissable que l'on peut situer au niveau vibratoire de la manifestation, est à la fois totalement immanente et absolument transcendante à l'exercice de la compréhension qui lie entre eux les êtres doués de cette capacité d'expression. Or, comme le précise Scholem, c'est * le caractère symbolique du langage qui détermine cette dimension *. Rapprochant cette dimension de celle qui est explorée dans les travaux de Benjamin, il nous rappelle que " dans le langage les mystiques découvrent une dignité, une dimension immanente, ou, comme on dirait aujourd'hui, une structure qui n'est pas vouée à communiquer ce qui peut l'être, mais bien au contraire, et c'est là le paradoxe de tout symbolisme, qui porte sur la communication d'un incommunicable dépourvu d'expression, et qui, même s'il en trouvait une, n'aurait en tout état 
de cause ni signification ni 'sens' communicable. $~^{18}$

De la même époque date vraisemblablement le " Fragment théologico-politique ", où Benjamin élabore une conception de l'histoire qui en fait non pas une catégorie de l'utopie, du royaume messianique, mais plutôt celle de * son approche la plus discrète *, qui s'instruit dans le caractère éternellement passager du bonheur, et correspond alors à l'éternité d'un déclin : "À la restitutio in integrum d'ordre spirituel, qui conduit à l'immortalité, correspond une restitutio temporelle qui conduit à l'éternité d'un déclin, et le rythme de ce temporel éternellement passager, totalement passager en sa totalité spatiale, mais aussi temporelle, est le bonheur. Car messianique est la nature de par son caractère éternellement et totalement passager. $w^{19}$

L'intégrité de la révélation qui commande l'approche du

${ }^{18}$ G. Scholem, * Der Name Gottes und die Sprachtheorie der Kabbala ^, (EranosJahrbuch, 39, 1970), pp. 243-299; trad. par Maurice R. Hayoun, in G. Scholem, Le Nom et les symboles de Dieu dans la mystigue juive (Paris, Cerf, 1983), pp. 5556. Sur l'évolution de la pensée de Scholem, voir l'excellente monographie de David Biale, Gershom Scholem. Kabbalah and Counter-History, seconde édition (Cambridge, Mass., Harvard University Press, 1982; version remaniée et abrégée de sa thèse parue aux mêmes éditions en 1979).

19 W. Benjamin, * Fragment théologico-politique \#, trad. par M. de Gandillac, Mythe et violence, p. 150. Dans la Préface du Trauerspielbuch, Benjamin fait valoir un concept d'origine qui procède de la même logique, donc où devenir et déclin, restitution et ouverture sur l'avenir cessent d'être perçus contradictoirement, obeissant alors à une rythmique bivalente nourrie par la dynamique des extrêmes (Origine..., pp. 43-44) : * L'origine, bien qu'étant une catégorie tout à fait historique, n'a pourtant rien à voir avec la genèse des choses. L'origine ne désigne pas le devenir de ce qui est né, mais bien ce qui est en train de naître dans le devenir et le déclin. L'origine est un tourbillon dans le fleuve du devenir, et elle entraîne dans son rythme la matière de ce qui est en train d'apparaître. L'origine ne se donne jamais à connâttre dans l'existence nue, évidente, du factuel, et sa rythmique ne peut être perçue que dans une double optique. Elle demande à être reconnue d'une part comme une restauration, une restitution, d'autre part comme quelque chose qui est par là même inachevé, toujours ouvert. $:$ 
royaume messianique n'est entrevue qu'à travers la diffraction de l'élément le plus éphémère, dans la dissémination d'une trace dont on ne peut recouvrer la source, puisqu'elle s'inscrit, pour nous prévaloir ici de la belle formule d'Emmanuel Lévinas, dans le sillage d'un " passé qui ne fut jamais présent ". De la même façon, les harmoniques du " pur langage * qui affleure dans les symétries brisées des langues livrées à leur propre sort, dans l'éclatement diasporique des formes d'expression où l'espèce humaine, le regard plongé dans l'encre de la nuit criblée de lueurs d'astres fossiles, tente d'apprivoiser l'ampleur de son abandon face au mutisme de l'espace sidéral, ne sauraient faire les frais d'une restitutio in integrum. A-t-on jamais accès à un * original », à l'editio princeps d'une cuvre, à la donnée immédiate d'une signification, à la "pureté " d'une intention, à une inscription vierge de toute altération?

Cette question est sans doute la plus pertinente et la plus promptement éludée en matière de traduction. Mais c'est aussi une question qui n'a laissé de hanter le travail de Jacques Derrida. Très tôt rompu aux enjeux de la traduction, y décelant le a passage à la philosophie ", et passé maître dans l'art de défiler les métaphores, il était inévitable qu'il rencontre le texte fameux de Benjamin, où ce dernier affürme entre autres que le recours aux idées de " vie * et de " survivance " pour caractériser le rapport intensif qui lie le sort de la traduction à celui des œuvres n'a rien de métaphorique (völlig unmetaphorischer). Déjà, en 1968, dans un entretien avec Julia Kristeva, il indiquait la nécessité d'en découdre avec l'imposition tacite d'un signifié transcendantal venant coiffer ou subsumer l'exercice de la traduction, en appelant alors à la notion d'une * transformation réglée d'une langue par une autre ${ }^{20}$, qui

\footnotetext{
${ }^{20}$ Jacques Derrida, * Sémiologie et grammatologie ", entretien avec Julia Kristeva, d'abord paru dans Information sur les sciences sociales, VII-3 (juin 1968), repris dans Positions. Entretiens avec Henri Ronse, Julia Kristeva, Jean-Louis Houdebine, Guy Scarpetta (Paris, Minuit, 1972), p. 31 : * Et c'est en effet dans l'horizon d'une traductibilité absolument pure, transparente et univoque, que s'est
} 
rappelle la mise au point de Benjamin, déjà citée plus haut, dans son essai de 1916, à savoir que la traduction * traverse des continuums de métamorphose, non pas des zones abstraites d'équivalence et de ressemblance."

L'ayant déjà maintes fois effleuré par des renvois obliques, ${ }^{21}$ c'est dans un texte publié en 1985, "Des tours de Babel ", que Derrida aborde plus directement l'essai de Benjamin'22. Son approche est non moins singulière que celle de Benjamin : alors que ce dernier met l'accent sur la " complémentarité * (Ergänzung) des langues, sur leur réconciliation dans la perspective d'une eschatologie messianique dont la matrice est le rapport intensif mesurant l'amplitude de leur différence à l'aune de la distance qui les sépare de la révélation, distance qui correspond à la mise en abyme de ce qu'il désigne comme le " pur langage * auquel elles aspirent secrètement, le propos de Derrida prend appel sur l'événement de Babel, où le détournement du nom, la tentative

constitué le thème d'un signifié transcendantal. Dans les limites où elle est possible, où du moins elle parât possible, la traduction pratique la différence entre signifié et signifiant. Mais, si cette différence n'est jamais pure, la traduction ne l'est pas davantage et, à la notion de traduction, il faudra substituer une notion de transformation : transformation réglée d'une langue par une autre, d'un texte par un autre. Nous n'aurons et $n$ 'avons en fait jamais eu affaire à quelque "transport" de signifiés purs que l'instrument - ou le "véhicule" - signifiant laisserait vierge et inentamé, d'une langue à l'autre, ou à l'intérieur d'une seule et même langue. "

21 Voir notamment * Living On - Borderlines „, trad. par James Hulbert, Deconstruction and Criticism, éd. par Harold Bloom et al. (New York, Seabury Press, 1979), pp. 75-176, version française dans Parages, Paris, Galilée, 1986; * Tables rondes sur la traduction *, L'oreille de l'autre. Otobiogrophies, transferts, traductions. Textes et débats avec Jacques Derrida, sous la direction de Claude Lévesque et Christie V. McDonald, (Montréal, VLB éditeur, 1982).

22 " Des tours de Babel *, première version publiée en 1985 dans Difference in Translation (Ithaca et Londres, Cornell University Press [édition bilingue]) et dans L'art des confins. Mélanges offerts à Maurice de Gandillac (Paris, PUF); repris dans Psyché. Inventions de l'autre (Paris, Galilée, 1987), pp. 203-235. 
d'usurpation et de monopolisation par la tribu des Schem ( nom * en hébreu) deviendra l'emblème de la confusion et de la dispersion des langues. La construction de la tour de Babel devient le levier de la déconstruction de l'utopie visant l'apanage de la langue unique, indivise. La lésion de la tour, son effondrement, s'inscrit dans la scission du nom propre Babel (ou Bavel), soit a la référence d'un signifiant pur à un existant singulier *, et son indexation comme nom commun dénotant la confusion. Le patronyme divin "Babel ", d'origine incontestée scellée dans le don du nom se fait source de division : il enraye la généalogie du nom propre, sème la confusion sur les lèvres de ceux qui, grand mal leur en prit, désirèrent se faire un nom. YHWH se plaît à perdre les zélateurs de la langue unique; tout espéranto lui répugne. Le nom propre sémitique " Babel " s'occulte en se généralisant, se traduit dans l'impropriété d'une signification qui devient commune aux usagers d'une langue où ils sont voués à ne plus s' $\alpha$ entendre ", à se disputer le sens du sens : le devenir-commun du nom, sa communauté, aliène celle de ses usagers - la " communauté inavouable " qui oblige à traduire à partir du lieu où cela s'avère impossible : le nom propre qui ne signifie rien, sauf qu'il n'a de propre que son don, s'exproprie, s'extrade, se sature d'un oubli qui agrée le fonctionnement de la langue, l'exercice du langage comme lieu de discrimination, articulation de la différence qui doit incessamment conjurer son propre " différer ", le reflux de la dissémination qui scelle son origine dans la contraction d'une dette sans acquit : " La traduction devient loi, le devoir et la dette mais de la dette on ne peut plus s'acquitter. Telle insolvabilité se trouve marquée à même le nom de Babel : qui à la fois se traduit et ne se traduit pas, appartient sans appartenir à une langue et s'endette auprès de lui-même d'une dette insolvable, auprès de lui-même comme autre. Telle serait la performance babélienne. " (Psyché, pp. 210-211)

Le mandat du traducteur obéit à la forme de la demande, qui est la forme où s'inscrit ce qui se donne à traduire. Ce qui signifie du même coup qu'elle transcende la disponibilité du traducteur, 
bref toute la gamme des trouvailles, des clefs heuristiques et des décisions pragmatiques qui sont du ressort de son exercice, puisqu'elle procède d'une loi immanente, intérieure à l'original, qui prévient l'oubli et qui le suscite à la fois, celle d'une survie qui ne répond à aucune attente : « Étrange dette, qui ne lie personne à personne. Si la structure de l'œuvre est "survie", la dette n'engage pas auprès d'un sujet-auteur présumé du texte original - la mort, ou le mortel, la mort du texte - mais à autre chose que représente la loi formelle dans l'immanence du texte original. * (ibid., p. 217)

I1 y va ici, selon Derrida, de l'origine même du contrat, du pacte, de l'alliance, qui fait de la traduction la condition de possibilité, en un sens quasi transcendantal, de tout commerce, de toute entente contractée entre parties : il y a des langues, non pas seulement le langage, mais des langues. Pour ouvrir une parenthèse, remarquons qu'il $\mathrm{y}$ a davantage qu'une simple connivence entre les opérations de la traduction et celles de l'économie : si l'espace frontalier où s'effectue le trading requiert le truchement d'agents de change, il en appelle aussi à la plasticité d'agents traducteurs qui doivent établir des " vases communicants " entre des aires de jeu différenciées par des échelles de valeurs enracinées dans l'usage le plus prosaïque des langues. Or, dans le cas qui nous occupe, soit l'exigence à laquelle Benjamin astreint le traducteur, ce dernier n'est pas d'abord destiné à assurer l'aval d'un tel négoce, à établir un régime d'équivalences réglées entre les instances contractantes, bref à « transporter tel ou tel contenu, à communiquer telle charge de sens mais à re-marquer l'affinité entre les langues, à exhiber sa propre possibilité " (Psyché, p. 220). Cette tentative, cette percée en direction de l'affinité (Verwandtschaft) secrète que les langues nourrissent dans leur disparité originaire, est essentiellement de nature proleptique. La traduction est pure prolepsis, pure virtualité, c'est-à-dire qu'elle " rend présente sur un mode seulement anticipateur, annonciateur, quasiment prophétique, une affinité qui n'est jamais présente dans cette présentation " (Ibid.). L'épiphanie d'une pareille affinité ne sera jamais intégrale : nulle parousie, nul dévoilement ne viendra 
clore le jeu d'endettement mutuel entre l'original et la traduction.

Cette éthique rédemptrice qui commande l'acte de traduction et l'oblige à faire son deuil de la visée idyllique s'attachant à la translation univoque entre une langue-source et une langue-cible, celle-ci étant chevillée par la contrainte de la préservation du sens (Enthaltung des Sinnes), n'a pour tout point d'incidence ou pierre de touche qu'un contact fugitif (flüchtig), la tangence infinitésimale où le sens et la forme communiquent et s'absolvent du même coup de toute détermination extérieure à cette intime et éphémère cohésion. Mais c'est là le sens originel du terme grec symbolon, que Benjamin évoque en recourant au motif kabbalistique du "vase brisé ", où le détail le plus infime dans le bris qui fait l'unicité du débris épouse son complément qui luimême doit s'ajointer à un autre fragment, et celui-ci à tel autre encore, pour ainsi figurer de proche en proche une unité d'ensemble qui ne doit son existence qu'à ce rapport intensif, cette logique discrète qui permet l'ajointement du dissemblable, à l'instar de l'errance qui commande l'approche de ce royaume a à la fois promis et interdit où les langues se réconcilieront et s'accompliront w.L'interdit du royaume tient à cet intouchable qui sollicite la traduction, l'assigne à sa maîtrise d'œuvre et lui signifie la distance même qui lui permettra d'accoucher d'une version qui, par un contraste décisif avec la matière de l'original, ne se laissera pas elle-même traduire.

Cette " inquiétante étrangeté " que l'on ressent par exemple à la lecture des traductions des tragédies de Sophocle par Hölderlin, est l'aura dans laquelle s'enveloppe la poussée cardinale qui, dans le rapport à l'étranger, délite la langue " maternelle * et l'ouvre en direction de la reine Sprache qui, loin d'en appeler à la restitution d'un état virginal de la langue, marque plutôt par son inviolabilité la co-originarité des visées implicites à l'exercice du langage dans la diversité des idiomes. C'est là le sens de l'affirmation énigmatique, à tout le moins problématique, qui noyaute l'argumentation de Benjamin et selon laquelle l'affinité supra- 
historique des langues tient au fait qu'en chacune d'elles, prise à chaque fois comme un tout, quelque chose est visé qui est d'emblée le même (in ihrer als ganzer jeweils eines und zwar dasselbe gemeint ist) et qu'aucune ne peut atteindre isolément. Quel est ce * quelque chose ", ce * même * qui n'affleure dans la traduction, à travers l'éclatement diasporique des langues, qu'en embrassant le spectre entier de leurs intentions mutuellement complémentaires? Ce que visent les langues en leur envergure respective et dans l'affinité intensive qu'elles nourrissent à leur insu et qu'est appelé à révéler le labeur de la traduction, que Benjamin compare à celui d'une parturiente, c'est, écrit Derrida, " la langue même comme événement babélien, une langue qui n'est pas la langue universelle au sens leibnizien, une langue qui n'est pas davantage la langue naturelle que chacune reste de son côté, c'est l'être-langue de la langue, la langue ou le langage en tant que tels, cette unité sans aucune identité à soi qui fait qu'il y a des langues, et que ce sont des langues " (Psyché, p. 232).

Cet être-langue de la langue ne peut émerger que dans l'expérience de la limite inhérente à chaque langue qui se découvre dans le rapport à l'étranger, là précisément où l'abîme entrouvert par l'éveil d'une différence plus intime et plus lointaine à la fois que celle qui permet l'articulation du sens dans le renvoi de signifiant à signifié libère ces harmoniques, cet accord à peine perceptible qui vibre dans la disparité même des langues et qui mesure, tant en ampleur qu'en intensité, la distance qui les sépare d'un terme incessamment différé, celui que Benjamin désigne comme le * terme messianique de leur histoire ». L'expérience de la traduction, ou l'épreuve de l'étranger, se traduit dans le * savoir de cette distance " qui se mesure au texte sacré comme paramètre absolu de la traductibilité, en ce qu'il commande et interdit la traduction. Dans ce cas - ici nous traduisons, nous traduisons Derrida traduisant Benjamin - , l'origine n'est plus ce qui depuis toujours précède, mais bien ce qui depuis toujours devance. Comme la loi, celle du texte sacré qui prescrit et interdit de traduire, montre la limite là même où elle se dérobe, nous investit 
et nous dévaste comme des voyelles qui dansent entre les consonnes. Allons plus loin : il y a traduction, matière à traduire, depuis que le monde est monde. Plus précisément, il y a monde, le monde est monde depuis qu'il y a traduction. Il y a monde depuis que l'être-langue de la langue s'est révélé dans le passage des frontières inscrites déjà dans la langue indigène confrontée à une altérité qu'elle ne peut apprivoiser, qu'elle ne peut approcher qu'en s'extradant vers sa propre limite. Cette altérité n'est pas celle de l'autre qui est inviolable (et en cela même toujours violée, ostracisée, pourchassée), mais la sienne propre qui, à se traduire en traduisant, n'en sort pas indemne, égare son certificat de virginité, car l'origine est devant soi, hors de soi, comme pure promesse, pure relation d'inconnu liée, comme l'écrit Benjamin, à la " survivance éternelle des cuvres " (am ewigen Fortleben der Werke) et à la a régénération infinie des langues " (am unendlichen Aufleben des Sprachen).

Dès lors, si le concept d'origine ou d'une " commune provenance » demeure indispensable pour Benjamin, il convient à notre sens de le saisir dans la perspective déjà indiquée dans le "Fragment théologico-politique " et dans la Préface du Trauerspielbuch. À savoir la double optique d'un devenir et d'un déclin, d'une croissance et d'une perte irrémédiable, bref une restauration n'ayant pour toute chance que l'ouverture à l'infini qui se dessine dans le frayage diasporique d'une * faible force messianique * dont le point de fuite s'inscrit dans le descendendo infini des langues. C'est par cette idée d'une " descente infinie des langues "que Giorgio Agamben se propose de cerner le lien profond, proprement inépuisable, qui unit le mouvement de l'histoire et le destin de la vie langagière chez Benjamin, dans sa communication au Colloque de Paris en $1983^{23}$. Les prémisses de

${ }^{23}$ Giorgio Agamben, * Langue et histoire. Catégories historiques et catégories linguistiques dans la pensée de Benjamin *, trad. de l'italien par Yves Hersant, Walter Benjamin et Paris, ed. par Heinz Wismann (Paris, Cerf, 1986), pp. 793807. 
son interprétation sont fournies par ce passage des notes préparatoires aux Thèses sur la philosophie de l'histoire qui, de l'avis d'Agamben, formule * en un lumineux raccourci, l'une des intuitions les plus profondes de Benjamin ". Qu'on nous permette donc ici de le citer dans son intégralité :

Le monde messianique est le monde de l'actualité totale et intégrale. Ce n'est qu'en lui qu'existe une histoire universelle. Ce qui est appelé aujourd'hui de ce nom, ne peut être qu'une sorte d'espéranto. Rien ne saurait lui correspondre tant que la confusion née de la tour de Babel subsiste. C'est qu'elle suppose une langue dans laquelle tout texte d'une langue vivante ou morte doit pouvoir être intégralement traduit. Ou mieux encore, elle est cette langue ellemême. Non comme langue écrite, mais comme la langue célébrée, fềtée. Cette fête est purifiée de toute cérémonie et ignore les chants. Sa langue est l'idée de la prose elle-même, qui est comprise de tous les hommes, comme la langue des oiseaux est comprise des enfants nés un dimanche. (G.S., I, 3, p. 1239; cité et trad. in * Langue et histoire ", p. 793)

Le propos de Benjamin est limpide, quoique déconcertant à la première lecture : l'idée d'une rédemption à l'échelle universelle est indissociable de l'assomption libératrice d'une langue absoute de toute contingence communicationnelle, de tout parasitage idiomatique, et cette lingua franca purement festive est l'a idée de la prose » elle-même, purifiée de tout protocole, bref des règles et des felicity conditions prescrites pour assurer la performativité des actes de langage liés à la transmission d'un contenu ou à l'atteinte d'un effet, d'une action escomptée de la part d'un destinataire. Nous élaborons évidemment, mais c'est bien cela que Benjamin désire écarter, dissoudre, cela qui se donne pour grammaire universelle et qui se résout dans une architectonique factice, une espèce d'espéranto. Il est surtout remarquable que la dimensiontémoin de cette émancipation soit celle du langage, alors que la littérature apocalyptique abonde plus volontiers dans le registre de la vision béate, comme si on n'en avait pas déjà assez vu. La donnée essentielle de cette projection, en effet, est que la félicité 
de la créature accédant au royaume messianique se traduit illico par la latitude inconditionnée d'une langue sevrée de toute codification, jouissant alors de cette " actualité totale et intégrale " qui agrée la traductibilité immédiate de toute langue, vivante ou morte. On pourra donc imaginer que la situation contraire, celle où la traduction s'impose et n'en finit plus d'œuvrer, est l'enfer, ou une bénédiction pour les fossoyeurs de Babel.

Pour le peu que chacun d'entre nous, et même ceux parmi les plus chevronnés en matière de langage, sait apprécier de la diversité des langues qui ornent l'œkoumène, il est difficile d'imaginer ce à quoi peut correspondre " une langue dans laquelle tout texte d'une langue vivante ou morte doit pouvoir être intégralement traduit ". La barre est haute. Ou plutôt convient-il d'envisager cette utopie du tréfonds de la vie langagière, à l'autre bout du spectre défini par la " descente infinie des langues ", qui n'est autre que la physionomie subtile de l'histoire comme ars grammatica. Servi par une vaste érudition, Agamben esquisse une brève généalogie de cette conception qui trouve son fondement dans la réflexion stoïcienne sur le langage; ainsi, le grand traité de Varron sur la langue latine, d'inspiration stö̈cienne, distingue deux plans du langage, celui des noms (de la pure nomination), et celui du discours, qui en dérive " comme une rivière de sa source " (De lingua latina, VIII, 5-6; cité et trad. in " Langue et histoire ", p. 794). Tout acte de langage présuppose déjà le plan des noms, le "don de la langue " qui se prête tant et si bien à l'oubli des générations, à l'amnésie du système de référence qui dessert, dans les deux sens du terme, le " sens commun ", qu'il resurgit comme fonds intarissable donnant matière à une recréation qu'on estimera volontiers être le fruit d'une " génération spontanée ". Dante Alighieri s'en était déjà avisé. Dans le Convivio, en effet, il compare la grammaire au ciel de la lune, à cause de a l'ombre qui en celle-ci apparaît, laquelle n'est autre chose que rareté de sa matière, à quoi ne se peuvent terminer les rais du soleil et se refrapper comme dans les autres parties $n$; or c'est là une propriété que possède la grammaire, nous dit Dante, car « du fait qu'elle est 
non finie, les rayons de la raison n'y trouvent pas de terme, spécialement en matière de vocabulaire; et elle luit ore de ça, ore de la, en ce que certains vocables, certaines déclinaisons, certaines constructions sont en usage, qui jadis n'étaient point; et beaucoup furent jadis, qui de nouveau seront : comme dit Horace au début de la Poetria, quand il dit : "Maints vocables renaîtront, qui jadis tombèrent" $n^{24}$.

Il en ressort que la raison instrumentale ou logicogrammaticale ne saurait fonder et encore moins atteindre le fond, tarir le fonds des noms, de la nomination, car la dissémination ou, pour emprunter une image de Novalis, la pollinisation des vocaboli est fonction d'un " descendendo " infini qui est l'histoire comme telle. L'oubli du nom dans son usage le plus prosaïque comme dans sa forme la plus épurée, la plus affranchie de sa tangente communicationnelle, se résout dans une condition débitrice qui ne peut épuiser son passif à l'endroit d'un passé immémorial dont le ressourcement ne laisse de se différer, de s'extrader * vers le futur d'un courant infiniment descendant de sorte que la pensée ne peut jamais trouver en lui son point d'aboutissement. * Or c'est là, poursuit Agamben, “ l'"ombre" irrémédiable de la grammaire, l'obscurité qui s'attache originellement à la langue et qui fonde dans la nécessaire coïncidence de l'histoire et de la grammaire la condition historique de l'homme. L'histoire est le chiffre de cette ombre qui voile l'accès de l'homme au plan des noms : l'histoire est à la place des noms. La transparence du langage l'absence de fondement de tout acte de parole - fonde ensemble la théologie et l'histoire. Tant que l'homme ne pourra trouver dans le langage un fondement, il y aura transmission des noms; et tant qu'il y aura transmission des noms, il y aura histoire et destin ("Langue et histoire ", pp. 795-796). Agamben en tire donc cette conséquence ultime qui en réalité se veut la condition originaire de

${ }^{24}$ Dante, Banquet (Convivio), II, XIII, 8-10, trad. par André Pézard, Oeuvres complètes (Paris, Gallimard, Bibliothèque de la Pléiade, 1965), p. 349. 
l'homme, le mortel : a La condition historique de l'homme est inséparable de sa condition d'être parlant et se trouve inscrite dans la modalité même de son accès au langage, originellement marquée par une scission " (lbid., p. 796).

C'est cette scission précisément que Benjamin a désiré marquer dans son essai de 1916 comme la chute de l'homme hors du règne de la " langue des noms ", laquelle " ne connaît aucun moyen, aucun objet ni aucun destinataire de la communication ", encourant alors une inhibition qui se traduit par la syncope de l'expression dans la forme prédicative du jugement et la conceptualisation de la dimension symbolique qui se voit de la sorte refoulée dans les limbes de l'abstraction d'où la traduction doit l'en tirer par un véritable travail d'obstétrique, suivant les termes de l'analyse de Benjamin. Ce travail consiste à affranchir des liens de la signification, d'une conception de la langue comme système de signes véhiculant des signifiés, ce qui serait déjà " entendu " en toute langue historique, ce à quoi chacune aspire et qui repose sur le paradoxe constitutif de la théorie du langage chez Benjamin, à savoir que " toutes les langues veulent dire le mot qui ne veut pas dire " (" Langue et histoire ", p. 798).

C'est l'exigence à laquelle Benjamin lui-même aura plié sa propre écriture, bien que celle-ci ait souvent prêté le flanc aux critiques la taxant d'ésotérisme. Le réseau complexe de ses analyses en matière de langage vise une intuition directe de son essence qui, pour être acquise à un messianisme pleinement sécularisé qui ne saurait faire l'économie de l'histoire, ne se laisse pas réduire à une simple composante de sa trame narrative. La position que Benjamin assume à cet égard ne saurait donc être conciliée à celle de l'herméneutique contemporaine, selon laquelle tout acte de parole recèle un non-dit qui se prête à une infinité de sens et confirme ainsi la finitude du discours humain et l'ascendant historique d'une tradition qui en appelle à une communauté idéale d'interprétation, sinon à ce que Heidegger désigne comme le " cercle herméneutique ", ou encore, dans les termes de Gadamer, 
à une " fusion des horizons " dans lesquels se projette toute quête de sens. Pour Benjamin, c'est la rupture de l'histoire, l'interruption d'une narrativité rompue aux " ruses de la raison " (Hegel) et largement insensible à l'oppression qu'elle cautionne, qui seule peut donner lieu à une épiphanie du sens où se cristallise l'hyperbole catastrophique de l'histoire, qui la révèle dans son ampleur et dans son intensité, mais uniquement l'espace d'un instant qui préfigure l'accès au royaume messianique. Mais le propos de Benjamin, dont il n'est guère besoin de souligner l'extrême singularité, se démarque aussi des tenants de l'orthodoxie kabbalistique, du prestige de la lettre où l'on cultive la synergie ou la symbiose de l'essence spirituelle et de la combinatoire des dénominations visant l'u être littéral » du Nom divin : " Alors que le caractère mystique et in-signifiant du nom de Dieu est lié, dans la Kabbale, à son être purement littéral, Benjamin affirme explicitement que la langue de l'humanité rédimée a "brisé les chaînes de l'écriture" et qu'elle est une langue "non écrite, mais célébrée dans l'allégresse". À la démarche des kabbalistes, pour qui s'écrit ce qui n'a jamais été dit, s'oppose ici une "lecture de ce qui n'a jamais été écrit" " "Langue et histoire ", p. 803). Autrement dit, non seulement Benjamin prend acte d'un retrait définitif du Nom divin hors de la sphère du dicible, mais le sens et la portée de ce retrait échappent désormais à toute codification, puisqu'il répond à une cassure irréversible. C'est la trace de ce retrait qui affleure dans le labeur de la traduction, comme point de fuite inscrit dans l'interrègne où se consomme la perte définitive de la "langue des noms " et se dessine la possibilité d'une croissance intensive des germes du " pur langage * disséminés dans la diaspora des langues.

La " langue des noms * ne saurait donc être assimilée à un stade initial du devenir des langues, pas plus que le " terme messianique de leur histoire " ne coïncide avec la parousie d'une langue idéale, marquant la fin de l'exil de la parole dans la prolifération post-babélienne des idiomes. Le point de convergence de la pré- et de la post-histoire (Vor- und Nachgeschichte) qui, 
suivant l'analyse de la Préface de l'étude sur le Trauerspiel, peut seul faire droit à un concept d'origine purgé de toute nostalgie comme de toute attente apocalyptique, est l'" idée de la prose ", qui correspondrait alors à une pure lingua franca, littéralement une " langue affranchie " de tout référent extérieur à sa manifestation, de toute contrainte liée à sa * mise en scène ", à commencer par le clivage entre la forme et le contenu. La fin de l'exil de la parole correspondrait plutôt à la jouissance de la traductibilité absolue, immédiate, de l'expression gratifiée par une " actualité intégrale " que Benjamin qualifie aussi de " allseitig ", c'est-à-dire qu'elle embrasse de toutes parts le champ de la manifestation (G.S., I, 3, p. 1239). L'" idée de la prose " est pure exposition, elle ne présuppose (voraussetzen) rien; elle marque plutôt l'accès à l'êtrelangue de la langue comme pur médium, comme mi-lieu.

Sans doute est-il juste de saisir dans cette poussée cardinale vers une langue affranchie de toute division topique, c'est-à-dire désormais ouverte à l'instar d'une pure chôr $a^{25}$, une matrice sans frontières, la vérité même de l'exode de la créature, à savoir l'exigence inconditionnelle d'un déracinement constant face au rapt des forces titano-telluriques qui rivent l'homme à un sol, à un horizon qui le retient captif sous le joug du mythe qui condamne à la répétition stérile de l'acte sacrificiel. Que le lien infrangible qui scelle le destin de l'homme dans son rapport au langage soit la pierre de touche de cette tension messianique ne saurait étonner; c'est en lui que s'est joué le désenchantement du monde mythique, et c'est aussi en lui que s'est abolie toute possibilité de a tenir parole " dans la postérité d'Auschwitz. Ce rapport au langage est l'indice d'une rupture qui, au-delà de la rigueur souveraine du logos grec, nous reporte ultimement à l'éveil de la judéité en l'homme, à ce que Massimo Cacciari désigne dans ses Icônes de

${ }^{25}$ Sur cette notion hybride, indéfinissable et pratiquement intraduisible, qui apparaît dans le mythe cosmologique du Timée de Platon et dont ce dernier dit qu'elle n'est ni * sensible $* \mathrm{ni}$ " intelligible " mais appartient à un "troisième genre ^(triton genos, 48e, 52a), voir J. Derrida, Khôra (Paris, Galilée, 1993). 
la loi comme la " Racine errante ${ }^{26}$ qui traverse les langues et les terroirs, qui les féconde sans jamais s'y laisser enfermer, car n'ayant d'autre vertu que de radicaliser le rappel à la condition

${ }^{26}$ Massimo Cacciari, Icônes de la loi, trad. par Marilène Raiola (Paris, Christian Bourgois, 1990); ici nous ne pouvons que citer in exfenso ce passage remarquable qui jette un tout autre éclairage sur ce à quoi Benjamin peut référer dans son évocation d'un * pur langage * (op. cit,, pp. 47-48) : * Tout comme le peuple de la vie éternelle est partout étranger, car sa racine lui impose de reconnaîttre comme patrie authentique uniquement une terre sainte, donc constamment autre que celle où il vit, il traverse les différentes langues, sans jamais se confondre avec elles. Tout comme sa racine est le sens d'une nostalgie inexorable, sa langue n'est jamais maternelle, la langue qu'il parle n'est jamais la sienne. Il parle toujours dans une langue étrangère; depuis des siècles sa Langue vraie, sa Langue éternelle a cessé de "servir" le quotidien. Elle s'est transformée, pour ainsi dire, en la Langue de cette racine, en témoignage-anticipation du futur éternel. Mais témoignage ici-et-maintenant et, donc, capable d'intervenir dans le cours "domestique" des langues, de les transfigurer à travers des souvenirs, vestiges, citations de son Uberwelt. Un réseau inextricable de citations-traductions rattache la Langue aux Langues; un système vivant de canaux, de courants, de parcours montre l'éternelle vie de la Langue, dans les langues et la nostalgie étemelle de ces dernières pour celle-là. Ce feu est présent dans chaque texte - qui plus est dans chaque signe, dans chaque écriture : la "rupture des vases" et les misères du "retour" existent en chaque mot. Dans la langue quotidienne elle-même, la simple présence de la citation suffit pour que ce feu se ravive; là se manifeste comme un reflet de son secret : là se crée un tourbillon qui l'arrache à la nostalgie de la Langue; là se montre cette racine qui déracine, qui fonde l'errance. Cette citation fait apparaître tout discours comme une douloureuse et pénible traduction, elle arrache de celui-ci toute apparence "naturelle", "maternelle". L'abîme de l'aitia, de l'arché s'offre dans sa béance. Aucun mot ne semble plus pouvoir désigner directement - seule cette aitia paraît le rendre signifiant, cette aitia qui renvoie, de fondement en fondement, à l'imprononçable, au silence de l'origine. La Langue n'est pas une langue morte, elle ne se situe pas dans une dimension abstraitement autre par rapport à celle des langues. C'est la force qui les rend inquiètes, jamais chez soi, un-heimlich, en chacune de leur époque; c'est la force qui empêche de se fier à elles, d'être en harmonie avec elles. La Langue est ainsi la seule vraiment vivante, puisqu'elle exile de la dimension mortelle des langues et de toute foi idolâtre en leur pouvoir. La langue est donc, ainsi, la parole même de l'exil. Si elle n'habitait pas près de nous c'est à peine si nous pourrions le reconnaître comme tel. Ces terres seraient les nôtres, ces langues seraient maternelles - si des souvenirs, des citations, des traductions de la Langue n'y faisaient pas continuellement irruption. . 
migratoire de la créature, qui seule peut rencontrer l'impératif d'une sanctification du temps comme vecteur de la rédemption de l'état de nature - une nature livrée au mutisme des origines, mais encore à ce silence impénétrable qui transit de sa sourde étreinte le microcosme tragique, comme une transcendance foudroyée sur son céans. Cacciari y décèle au passage le lien étroit qui unit la pensée de Benjamin et celle de Franz Rosenzweig dans leur attachement respectif au travail de la traduction, de la citation et du commentaire ${ }^{27}$. Dans ce cas, la traduction, loin seulement de se voir confinée à une fonction ancillaire qui consiste à ménager un relais entre les langues en y escomptant un minimum d'entropie du signifié, témoignerait d'une exigence beaucoup plus profonde qui prend acte en toute lucidité de la dimension tragique de l'existence humaine et pratique une brèche, une déchirure dans l'horizon

${ }^{27}$ Franz Rosenzweig (1886-1929), l'auteur de l'Éfoile de la Rédemption (trad. par Alexandre Derczansky et Jean Louis Schlegel, Paris, Seuil, 1982), sans conteste l'une des œuvres majeures de la pensée philosophique au XX' siècle, $\mathrm{s}^{\prime}$ était par ailleurs engagé avec Martin Buber, à partir de 1925, dans un projet de traduction intégrale en allemand de la Bible hébralqque. Il a aussi publié de remarquables essais sur le concept et la pratique de la traduction, dont certains passages trahissent une nette affinité avec la conception de Benjamin; la plupart de ces essais ont été recueillis avec ceux de Martin Buber dans un volume paru en 1936, Die Schrift und ihre Verdeutechung (Berlin, Schocken Verlag), et qui a été récemment traduit en anglais, dans une version augmentée et annotée, par Lawrence Rosenwald et Everett Fox, Scripture and Translation (Bloomington, Indiana University Press, 1994). On pourra notamment consulter $\propto$ Die Schrift und Luther », paru en 1926 (trad. citée, pp. 47-69). Mais là où le propos de Rosenzweig s'avère le plus proche de la pensée de Benjamin, c'est dans l'essai qui accompagne ses traductions de Yéhuda Halévi, parues en 1924, où il affirme entre autres qu'il y a une langue unique et que chacune des langues mobilisées par l'être humain y est contenue à l'état d'embryon, de la même façon que toute particularité affichée par une langue donnée est aussi contenue à l'état embryonnaire dans n'importe quelle autre langue, de sorte que l'impératif qui gouverne l'exercice de la traduction procède de cette unité essentielle qui en recèle à la fois la nécessité et la possibilité; voir F. Rosenzweig, Jehuda Halevi. Füfundneunzig Hymnen und Gedichte. Deutsch und Hebrdisch mit einem Vorwort und mit Anmerkungen, éd. par Rafael N. Rosenzweig (La Haye, Martinus Nijhoff, 1984), p. 3. 
auquel est rivé le regard scotomisé des peuples emmurés dans leur autochtonie.

Que le rapport au langage soit empreint d'une " tonalité * tragique, que celle-ci soit liée à la dissymétrie violente des langues qui s'affrontent dans une arène contaminée par l'effet de " surdénomination ", par l'inflation prohibitoire d'un réseau de signifiés (la logique procédurale par exemple) se résolvant dans l'absence de fondement qui caractérise l'origine mythique du droit, c'est là l'une des conclusions vers lesquelles s'acheminait Benjamin dans son essai de 1916. C'est aussi par ce biais qu'Alexander Garcia Düttmann aborde et approfondit, dans la Parole donnée ${ }^{28}$, la problématisation de l'essence du langage chez Benjamin. Suivant le scénario de l'essai de 1916, la condition postbabélienne de la vie langagière, la démultiplication protéenne des idiomes, en cela même qu'elle touche à l'essence spirituelle de l'homme, correspond à l'exil de la parole hors de la condition paradisiaque de la Namensprache dans laquelle était encryptée la puissance créatrice du verbe divin. Même le mutisme originel des autres créatures, auparavant appelées à jouir de la félicité de la dénomination adamique, est affecté par cette désaffection, ce " détournement" (Abkehr) de la langue des noms : une autre " mutité " (Stummheit) s'instaure, qui trahit la profonde tristesse (Traurigkeit) de la nature, sa mélancolie. Or, comme Benjamin tient à le stipuler, ce n'est pas parce qu'elle est muette qu'elle est triste, c'est plutôt la tristesse de la nature qui l'amène à s'enfermer dans son mutisme. Comme le note à son tour Düttmann, cette distinction s'impose parce que " le langage des noms qui traduit le langage des choses perd la force de nommer, de traduire en nommant : il devient le langage des signes arbitraires. Une différence de degré sépare la béatitude propre à l'homme dont la

2s Alexander Garcia Düttmann, la Parole donnée. Mémoire et promesse (Paris, Galilée, 1989). 
vie s'accorde avec l' "esprit langagier pur" (reiner Sprachgeist) de la béatitude qu'il faut attribuer à la nature muette et non mélancolique. Mais aucune différence de degré n'est repérable dans la mutité : elle se sépare d'elle-même par une rupture. " Autrement dit, poursuit Düttmann, " la perte du langage des choses, l'impossibilité de traduire la chose implique une perte de sa mutité essentielle et originaire. La mutité est à la fois le trait décisif du langage des choses, de la chose qui reçoit le nom qui lui correspond, et l'effet de la mélancolie " (la Parole donnée, p. 112). Ainsi, la Sprachlosigkeit, la privation du langage, n'est pas seulement la perte d'un actif virtuel, mais l'instauration généralisée de l'empire du deuil, de la mélancolie et de l'oubli qui s'étend à l'ensemble du royaume créaturial, " Dans toute forme d'affliction, écrit Benjamin, est très profondément ancrée la tendance à perdre la jouissance du langage, et ce deuil est infiniment plus qu'une inaptitude ou une répugnance à la communication. Ce qui est affligé se sent transi de part en part par la connaissance de l'inconnaissable. Être gratifié d'un nom même si celui qui donne ce nom est l'égal des dieux et comblé de félicité - demeure peut-être toujours un présage de tristesse * (Mythe et violence, p. 96; trad. modifiée).

I1 y a donc un abîme que jamais on ne saura combler, puisqu'il répond à une blessure insuturable, un abîme donc entre la mélancolie originaire qui n'est autre que l'affect de la nature sensible face à la défection du nom, et le règne de la surdénomination liée à l'arbitraire des signes. Mais, comme le précise Düttmann, il ne s'agit pas là d'une carence, d'un manque, d'un défaut. Ce qui est inappropriable dans le nom, ce qui se prête à l'oubli et demeure en cela même inoubliable, c'est * le langage lui-même, ou plutôt son don, ce don qui s'efface dès qu'il apparaît " (la Parole donnée, p. 120). Le " don du langage ", la Gabe der Sprache qui assignait l'homme comme celui devant concevoir (empfangen) dans le nom " ce qui est sans nom " (das Namenlose), encourt une lésion, une schize inhibitoire qui procède de la douleur immanente à l'appel de la créature qui se mure dans 
l'incommunicable, donnant lieu à la pure " monstration * du signe orphelin du nom, bref à la déshérence d'un don qui se diffère en prescrivant sa propre oblitération. " Le mutisme, écrit Düttmann, ce mutisme qui est le fond enfoui de la plainte situe donc la chose au sein même du langage - des noms : la chose prescrit la surdénomination dont elle se plaint. Elle se révèle être l'inappropriable ou l'incommunicable, ce qui se montre sans se montrer dans la mélancolie. n (lbid., p. 121)

L'élément de la signification dans le langage est l'apanage du tragique, alors que la langue des noms est une signification sans signification, n'appartenant comme telle ni à l'ordre de la nécessité, à la forme du jugement, ni à l'empire de l'arbitraire et de l'aléatoire. La surdétermination qui règne dans la relation tragique entre les langues provient de l'individuation de la forme qui est vouée à la signification, à la totalisation d'une finitude close sur elle-même et ainsi soumise à "l'éternelle rigidité du verbe proféré ". Or l'ironie majeure qui constitue le paradoxe de l'individuation tragique est que le héros " meurt d'immortalité ", tout comme le nom, en s'aliénant au profit de la signification conférée par la forme du jugement, s'emmure dans l'abstraction, " tombe " sous le concept, sous la juridiction de l'universel.

Cette ironie domine le rapport à la langue dite " maternelle ", qu'on ne possède qu'en y étant dépossédé, qu'en éprouvant sa limite dans la traduction, c'est-à-dire l'ambiguitté du rapport à l'étranger qui est encrypté en elle, qui la délite, l'extrade vers une limite qu'elle doit se dissimuler pour fonctionner. C'est ce que Catherine Mavrivakis désigne comme la " crypte de la langue ", à savoir " le lieu mythique de cet absolu d'un désir de pureté qui n'existe que dans sa traduction $w^{29}$. Il n'y a pas de langue originelle, toute généalogie est enrayée. Mavrivakis évoque le fiction

${ }^{29}$ Catherine Mavrikakis, \& la Traduction de la langue pure : fondation de la littératurew (TTR, vol. 2, n 1, 1989), pp. 59-74. 
théorique de Mallarmé dans les Mots anglais. Ce dernier y invente littéralement le paradigme de sa poétique, l'archéologie fictive de sa propre extradition hors de la langue normative, courante, qui n'existe pas davantage que sa subversion : sa positivité est d'ores et déjà mise en abyme dans la traduction qu'elle sollicite pour préserver la crypte où elle entretient le fantasme de sa pureté. D'où, en l'occurrence, "l'idée d'une crypte originaire de la langue où la langue serait intraduisible, inatteignable, pure et jamais contaminée par celui qui la parle : elle est construite dans l'espace même de la langue et ne peut célébrer aucune antériorité originelle. Pourtant, à travers cette fiction d'une résistance de la langue à ellemême, la langue est déplacée vers le lieu de son absence, de son non-sens et de sa disparition. Cette crypte mythique de la langue pure, il ne faut pas y toucher, ne pas l'ouvrir sous peine de perdre la langue. Elle renferme le vide de tout langage $\times(1989$, p. 70$)$.

Ce vacuum à l'intérieur de ce qui devrait être le plus propre à l'usage d'une langue dite a maternelle " est précisément ce qui est investi par la traduction, en ce qu'elle est appelée à faire l'expérience dans le nom de ce qui est " sans nom ". Le " noyau pur " du langage qui, selon Benjamin, devrait être visé par toute traduction, du moins celle qui répond à l'impératif de l'Umdichtung, bref l'appel à une transmutation de la poétique de l'original, ce noyau pur du langage n'existe pas, il n'adhère à aucune construction finie de la langue; il n'est autre que ce qui se diffère sans fin et oblige comme tel à re-marquer le nom - le don de la langue qui commande son propre oubli, qui rançonne de sa propre amnésie le pacte qui sanctionne son indexation au régime cognitif qui gouverne le fonctionnement du langage comme instrument de communication. La méconnaissance du nom est le levier d'une connaissance qui ne traduit pas la chose mais la juge $\mathrm{j}^{30}$.

\footnotetext{
${ }^{30}$ C'est le point de vue qui est assumé de plein droit par Gottlob Frege (18481925) dans son projet d'épuration logiciste visant à restituer les lois de la pensée. Il est notamment connu - le spectre de ses recherches est beaucoup plus vaste pour sa distinction entre * sens "et * référence *. Dans un article célèbre paru en
} 
Si l'incommunicable qui correspond à la communicabilité même de la langue est la matière première de la traduction, cette dernière est le lieu même d'une suspension du jugement, d'une épochè radicale en regard de l'intentionnalité inhérente à l'expression comme véhicule de sens. Ce hiatus abyssal dans l'articulation du signifiant et du signifié répond à l'impossibilité de s'approprier l'impropriété de ce qui est le plus propre à une langue qui ne peut se révéler à elle-même que dans le rapport à l'étranger, un étranger sur lequel elle ne peut porter aucun jugement. Comment pourraisje juger la langue cassée de Paul Celan à partir de mon incapacité à la traduire sans déjà lui faire violence? $\mathrm{Si}$, comme l'écrit Mavrikakis, « pour Benjamin la traduction n'est qu'une sorte de manière provisoire d'atteindre le noyau de la langue, il n'en reste pas moins que ce noyau ne se donne pas à connaître et que ce provisoire n'est en fait que le mouvement incessant de la création. La traduction n'est plus une particularité de la langue, ou une

1892, "Über Sinn und Bedeutung " (trad. française par Claude Imbert, in G. Frege, Écrits logiques et philosophiques, Paris, Seuil, 1971, pp. 102-126), il procède à la décomposition de la notion préalable de * contenu jugeable ", qu'il applique alors à ce qu'il désigne comme une * expression saturée ", où il distingue, d'une part, les noms propres, qui désignent un objet (par contraste avec les noms communs, qui reférent à un concept), et, de l'autre, les propositions. (Les expressions insaturées sont des signes des fonctions.) Les noms propres sont analysés en termes de sens, correspondant au mode de donation de la référence, et de référence, qui correspond à l'objet désigné. Les propositions sont analysées en termes de pensée et de valeur de vérité, et elles sont alors assimilées à des noms propres dont l'objet (logique) est la valeur de vérité, le vrai ou le faux : " Toute proposition affirmative, quand on considère la référence des mots qui la constituent, doit donc être prise comme un nom propre; sa reférence si elle existe est le vraj ou le faux $n$ (trad. citée, p. 110). Il va de soi, sous de tels auspices, que le domaine de la connaissance devra réserver ses attentions à la validation de la référence plutôt qu'à son mode de donation; autrement dit, une expression douée de sens ne pourra être réputée valide que si on peut lui octroyer une valeur de vérité. La traduction échappe à ce programme, puisqu'on ne dit jamais d'une traduction qu'elle est vraie ou fausse, mais qu'elle est bonne ou mauvaise. C'est ce que Benjamin exprime en toutes lettres lorsqu'il demande de considérer d'abord le a mode de la visée n (die Art des Meinens) plutôt que l'objet visé (das Gemeinte). 
quelconque opération linguistique, elle est la langue elle-même et la condition de son existence inscrite en elle. Elle affecte ainsi la langue non seulement dans son rapport interlinguistique, mais encore dans ses fondements intralinguistiques. La traduction serait alors le nom par lequel la langue se tait et parle sans pouvoir arrêter le mouvement. Sachant cela, il n'est plus souhaitable de tenter de dire la crypte dans sa pureté, car elle n'existe que dans sa traduction infinie et contient en elle ce qui n'est peut-être qu'un mythe, la totalité de tous les mots du monde n. (lbid., p. 72)

La traduction correspond à ce moment spécifique dans la vie intérieure du langage où ce qui est déjà nommé, archivé, déposé dans la langue, apparaît comme quelque chose qui n'a pas encore de nom, qui est " ignoré sous son nom ". Dans ce sens, il n'a rien de spécifique, il serait plutôt la marque de l'a-topique, du non-lieu, de l'extra-territorialité même de la langue restituée à une origine qu'elle ne saurait elle-même fonder. Nous irions plus loin : ce qu'une conception extensionnaliste du langage aurait tendance à étiqueter comme des cas d'* opacité référentielle ", comme spécimens affichant un haut degré de résistance dans le transfert d'une langue-source vers une langue-cible, loin d'être une espèce de marqueur occulte instaurant une zone grise dans la transparence souhaitée en matière d'expression, est l'indicatif même du lieu radical de la traduction comme matrice appelée à révéler l'êtrelangue de la langue, donc à communiquer cela même qui ne se communique pas : la communicabilité.

Ce qui dans le nom ne peut se traduire, c'est cela même qui commande la traduction, cet appel ultime qui est aussi le véritable appel du langage, le pur * dire " qui n'est pas fixé dans le * dit ". Dans une étude très étoffée où elle esquisse un rapprochement entre Heidegger et Benjamin, Éliane Escoubas ${ }^{31}$ voit dans leur

"Éliane Escoubas, * De la traduction comme "origine" des langues : Heidegger et Benjamin * (les Temps modernes, $n^{\circ}$ 514-515, mai-juin 1989), pp. 97-142. 
traitement respectif de la problématique de la traduction une approche intransigeante du noud de la question où le primat de la référence, ou encore la thèse de l'intentionnalité comme moment inaugural du * penser », sont disqualifiés; il s'agit plutôt de penser la traduction comme essence même de la langue dans son origine plurielle, marquée par le double jeu de la traductibilité et de l'intraductibilité qui a fait d'une langue une langue, la distinguant d'un code ou d'un chiffre ". (Escoubas, [1989], p. 98)

Chez Heidegger, notamment à partir de 1935, le nom même de * traduction * (Übersetzung) prend le relais du travail de la " répétition " (Wiederholung) de l'histoire de l'être, en appelant alors à une " destruction de l'ontologie ", une "déconstruction * (Abbau) où la traduction * nomme à la fois l'impensé de l'histoire de l'ontologie et le mode même de cette histoire " (lbid., p. 99). Pour ce qui est de Benjamin, Escoubas considère que ce sont les apories de l'essai de 1916 qui ont entraîné le déplacement de la question de l'essence du langage sur le terrain de la traduction. Ce déplacement est marqué par une pensée de la Dichtung et l'élaboration d'une théorie critique ayant pour horizon le développement d'une pensée de l'histoire où la notion de temporalité devient opératoire et, de là, celle de la traduction. Il nous serait loisible de reconstituer l'intertexte qui, selon Escoubas, se tisse entre le texte de Heidegger et celui de Benjamin et que l'on peut ainsi schématiser dans ses grandes lignes : d'abord, l'originarité de la traduction qui révèle par le fait même l'autoréférentialité de la langue; celle-ci est d'emblée de constitution plurale, elle est affectée d'une altérité essentielle qui la situe déjà dans "l'entre-deux-langues "; d'où le paradoxe de la traductibilité et de l'intraductibilité par lequel on convient de la nonsubstitutivité des langues : traductibles par essence, les langues sont insubstituables; enfin, l'entrelacs de l'idiomatique et de l'ekstatique, soit le repli sur soi de l'idiome et son exposition à l'autre langue où se noue l'Aufgabe de la traduction, l'unicité de son opération comme subversion du principe d'identité, ou du régime de l'équivalence et de la substitution. 
Escoubas nous reporte à l'introduction du cours de Heidegger sur Parménide, prononcé au semestre d'hiver 1942-1943 ${ }^{32}$, où l'intelligence même du mot "Übersetzung " fait l'enjeu d'une investigation très serrée. L'analyse que nous propose Éliane Escoubas ne l'est pas moins, et nous ne pourrons dans ces quelques lignes rendre justice à la densité et à l'extrême minutie de son interprétation. Heidegger effectue une descente vers le noyau sémantique de l'articulation de über et de setzen. La démarquant de la dénotation courante, expresse, qui fait de la traduction le cas d'un transfert (Übertragung) d'une langue dans une autre, Heidegger note que " parler et dire sont en soi un traduire dont l'essence ne s'épuise nullement dans le fait que le mot traducteur et le mot traduit appartiennent à des langues différentes. Dans tout dialogue et tout monologue règne un traduire originaire (ursprïngliches Übersetzen) " (trad. Escoubas, [1989], p. 103). Mais ce traduire intra-linguistique n'est pas seulement l'affaire d'une substitution dans la tournure de l'expression, d'une " reformulation " (Umschreibung) au sens du rewording chez Jakobson. La mutation s'opère dans le " dire " lui-même qui est déjà trans-posé (übergesetz) dans un autre élément de la vérité, une autre constellation de la pensée, correspondant à l'ouverture d'un horizon où s'instruit une tout autre capacité de questionnement. La langue est conviée par elle-même à sa propre élucidation. C'est pourquoi la traduction de la langue propre, l'effort pour s'y traduire, est de loin beaucoup plus difficile que celle qui doit accueillir le vocable étranger.

Le verbe allemand übersetzen connaît ici un dédoublement : selon que l'accentuation porte sur " setzen » et que la particule "über " est inséparable, il désigne le passage (Übergang) d'une langue à une autre langue; si c'est la particule " über " qui est accentuée et se veut comme telle séparable, le terme " übersetzen "

${ }^{32}$ M. Heidegger, Parmenides, vol. 54 de la Gesamiausgabe (Frankfurt am Main, Vittorio Klostermann, 1982). 
dénote un franchissement, une trans-position qui entraine une métamorphose. Comme le note Escoubas, cette dernière accentuation désigne la metabolê antérieure à l'u expression ", au "vouloir-dire ", constituant en quelque sorte sa condition de possibilité. Ce métabolisme intrinsèque de la langue renvoie donc à la conception humboldtienne du langage, à l'energeia de la langue, à sa " force " (Kraft), la * traduction originaire * devenant alors synonyme d'une auto-production de la langue qui, comme telle, est antérieure à « tout passage de l'étranger au propre et du propre à l'étranger, ainsi qu'à tout passage du propre au figuré (Umschreibung)" (Ibid., p. 106). La traduction au sens dérivé selon Heidegger, mais première dans l'acception courante de ce terme, est hétérologique, sa règle est l'équivalence, et son principe, celui de l'identité : elle vise l'appropriation de l'étranger et l'expropriation du propre. La traduction originaire vise plutôt l'écart intime qui règne au sein même de l'élément natif de la langue, l'étrangeté même du propre, le trait duplice (Zwiefalt) qui lie le vocable à une histoire traversée de ruptures et de mutations. La traduction originaire se rapporte donc à l'intraductible, à l'élément qu'Escoubas désigne comme l'idiome et qu'elle distingue de la langue comme telle, en ce que l'un n'est pas coextensif à l'autre, ni l'une à l'autre. Le paradoxe constitutif de l'être-langue de la langue, de son essence, tient à sa double teneur, à savoir la différence intra-idiomatique qui produit une langue selon la règle de l'homologie, et la tension ekstatique, en vertu de laquelle * une langue n'est une langue qu'exposée à l'autre langue (exposée à la traduction) " (Ibid, p. 107). L'implication essentielle de cette double articulation qui reconnaît un décalage structural entre langue et idiome, donc la non-coextensivité de l'un à l'autre, ainsi que la cooriginarité du rapport à l'étranger au sein même de son propre élément, est la double thèse selon laquelle, d'une part, une langue comporte toujours plusieurs idiomes, et, de l'autre, un idiome peut traverser plusieurs langues (Ibid., p. 108).

Cette articulation nodale de la dynamique interne du langage est vérifiable à travers divers cas de figures. Escoubas relève trois 
formes spécifiques : le transfert (Übertragung), la reformulation (Umschreibung) et la réinterprétation (Umdeutung). Le transfert (Übertragung) caractérise le passage d'une langue à une autre; il met en cause un régime d'équivalence et de substitution qui suppose en principe l'indifférence du sens par rapport à la langue qui le profère; celle-ci est alors vue comme un support séparable qui ne saurait entamer l'intégrité du signifié qui doit sortir indemne de cette opération. C'est là l'épreuve de l'étranger, l'interaction du propre et de l'étranger. La reformulation (Umschreibumg) concerne le passage du propre au figuré, l'emprunt d'une diversité de tournures plus ou moins facultatives à l'intérieur d'une même langue qui est pareillement posée comme un support séparable et dont le tropisme agrée son assimilation à une thétorique généralisée tablant sur l'impropriété générale de la langue. La troisième figure, celle de la réinterprétation (Umdeutung), implique un " changement du domaine d'expérience » qui affecte le rapport au sens; Heidegger parle ici de "renversement "(Umschlag) et de " mutation " (Wandlung) touchant à l'essence même de la vérité: les vocables mobilisés dans des langues nettement différenciées sont posés comme équivalents alors que l'horizon dans lequel ils sont projetés encourt une transformation radicale qui relève de la différence herméneutique. Par exemple, il n'est pas du tout indifférent que le vocable grec (parménidien) Alêtheia soit traduit en latin par le terme veritas, ou encore le terme grec logos par celui de ratio. Qu'est-ce à dire sinon que l'Umdeutung fonde * une temporalité successive : une "histoire", fondation qui constitue ce que Heidegger désigne comme "l'événement proprement dit" (das eigentliche Ereignis) - l'événement de la mutation de l'essence de la vérité ». (Escoubas, [1989], p. 109)

La notion de * traduction " (Übersetzung) proprement dite, dont nous avons vu qu'elle admet une double accentuation, doit être saisie dans son intrication originaire avec ces modalités, qui ne peuvent alors faire les frais d'une simple juxtaposition, car leur fonction respective est tributaire d'un jeu d'oppositions qui déterminent l'orientation de la pensée. La première opposition 
relevée par Escoubas porte sur les préfixes über- et $u m$ - : alors que le préfixe über- dénote un déplacement, un transport, une délocalisation correspondant au mode de la phora grecque, le préfixe um- marque un changement, une altération qu'on peut assimiler au mode de l'alloiôsis grecque. Une double opposition régit le rapport des racines verbales setzen/tragen et schreiben/deuten. Le premier binôme verbal s'analyse respectivement dans la position (setzen) et l'élaboration au sein même de la langue d'accueil de l'expression adéquate, et, d'un autre côté, la déportation (tragen) et l'injection de la langue de l'original dans la langue d'accueil. Le second binôme verbal (schreiben/deuten) s'attache à la différence de la forme et du contenu, à la règle de l'écriture et au procès de la signification.

Comme le souligne Escoubas, l'élaboration de ces différences a pour enjeu la conception d'une langue indivise, qui se présente comme la critique achevée du chorismos platonicien, soit la séparation de la sphère de l'intelligible et du monde sensible. Heidegger récuse du même coup le métaphorique comme opérateur métaphysique ${ }^{33}$ validant la séparation du signe (Zeichen) et du sens. En fait, Heidegger rejette d'un seul et même trait une notion de la langue comme simple support d'un signifié, sa réduction à la couche sémiotique de l'expression, et aussi bien le conventionnalisme lié à l'arbitraire du signe que le substantialisme sous-jacent au postulat de la substitutivité des signifiants. Pour

${ }^{33}$ Dans son cours (1955-1956) sur le Principe de raison (Der Satz vom Grund), Heidegger affirme en effet (trad. par André Préau, préface de J. Beaufret, Paris, Gallimard, 1962, p. 126) que " la notion de "transposition" et de métaphore repose sur la distinction, pour ne pas dire la séparation, du sensible et du non-sensible comme deux domaines subsistant chacun pour soi. Une pareille séparation ainsi établie entre le sensible et le non-sensible, entre le physique et le non-physique est un trait fondamental de ce qui s'appelle "métaphysique" et qui confêre à la pensée occidentale ses traits essentiels. "Heidegger en vient donc à ce diagnostic qui demanderait certes à être approfondi, en référence notamment à ce qu'en dit Benjamin lui-mêrne : * le métaphorique n'existe qu'à l'intérieur des frontières de la métaphysique $n$. 
Heidegger, la division des langues, qui sont elles-mêmes indivises, est celle de l'energeia, d'un jeu de forces dont le déploiement est assorti aux diverses constellations de l'histoire comme destin de l'être. Dans ce cas, du moins en ce qui touche aux " traductions essentielles n (wesentlichen Übersetzungen), celles qui, le temps venu, transfêrent (übertragen) une ceuvre de poésie ou de pensée, la traduction, lit-on dans le Principe de raison, n'est pas seulement " une interprétation (Auslegung), mais aussi une tradition (Überlieferung). En tant que tradition, elle appartient au mouvement le plus intime de l'histoire. Ce qui veut dire, d'après notre précédente remarque, qu'une traduction essentielle correspond toujours, à une époque du destin de l'être, à la manière dont une langue parle dans le destin de l'être ". (trad. remaniée par E. Escoubas, [1989], p. 112)

L'originarité du " traduire ", par contraste avec son usage dérivé, se rapporte à l'" historialité " (Geschichtlichkeit) de la langue dans sa différence intra-idiomatique, et celle-ci présente trois traits distinctifs selon Escoubas. D'abord, une seule et même langue est toujours en cours de traduction, elle est d'ores et déjà histoire, non pas au sens évolutif du terme, mais de par sa dimension ekstatique : elle est originairement structurée par son exposition à l'autre, qui détermine sa teneur " historiale ". Deuxièmement, le principe de la différence intra-idiomatique est le principe de la non-indifférence des langues : il n'est pas indifférent de parler telle ou telle langue. C'est ce qui fait du poète un traducteur originaire, un herméneute de la langue elle-même. Autrement dit, comme opérateur sui-référentiel de l'« historialité " de la langue, la différence intra-idiomatique est régie par l'homologie, qui n'est ni principe d'équivalence, ni principe de substitution-conservation. Enfin, les frontières des idiomes ne sont nullement les frontières des langues. Ainsi, Heidegger peut établir une espèce de bijection homologique entre le grec ionien des présocratiques et la langue allemande qu'il décortique suivant le procédé qu'il a fait sien au gré de ses inlassables ruminations. 
Le cas d'espèce sous examen, qui confirme la règle, est le vocable grec Alêtheia, qui appartient au lexique parménidien et qui sert de pierre de touche à l'élucidation de la mutation de l'essence de la vérité. Alêtheia est immédiatement traduit par Unverborgenheit, littéralement la non-dissimulation. C'est comme si ce terme était une citation du grec en allemand : le préfixe unn'est pas seulement privatif, mais dissociatif, il signifie une lutte contre la dissimulation. Or la langue grecque dispose de deux contraires pour le terme alêthes, soit lêthes (lathon) et pseudes. Il s'agit d'une reformulation (Umschreibung) à l'intérieur de la langue grecque par laquelle pseudes supplante lêthes. Cette bifurcation marque un écart qui entraîne l'occultation de Ia dimension de l'oubli contenue dans lêthes et l'instauration du règne de l'“ apparaître ", du régime de la " monstration ", du " porter-au-paraître " comme voilement inscrit dans l'usage du signe (la semiosis en l'occurrence) : * Le signe lui-même est un pseudos n, écrit Heidegger. Cette mutation endogène, donc licite du point de vue défendu par Heidegger, est signifiée par l'usage du préfixe non-négatif ent- au lieu du privatif un- : alêthes n'est plus traduit par unverborgene, mais par entborgene/entbergende où s'opère un redéploiement de l'essence du $a$ - de alêtheia, qui se voit ainsi servi par deux translats dans cette langue de prédilection que serait l'allemand: Autrement dit, comme le suggère Escoubas, un même idiome traverse le grec et l'allemand, ce dernier constituant la réserve de l'impensé de la langue grecque.

Mais une part d'ombre ne tarde pas à s'immiscer dans ces retrouvailles : en allemand, l'équivalent lexical du terme grec pseudos est das Falsche - le faux. Il n'est autre qu'un " calque " du terme latin falsum. Ce dernier procède de la racine verbale fallere, qui signifie tomber, " faire tomber $*-Z u$-Fall-Bringen selon le texte du cours sur Parménide ( $G A 54$, p. 60 ), et nous verrons plus loin que ce n'est pas du tout innocent. Ce spectre sémantique appartient de plein droit au domaine d'expérience de l'Imperium romanum. I1 s'agit cette fois d'une réinterprétation (Umdeutung) du grec pseudos, qui est contaminé par " quelque 
chose d'étranger ". Heidegger s'autorise du dictionnaire Grimm pour affirmer que das Falsche est un mot non allemand (undeutsches Wort). Cet import " fallacieux " du latin, l'intrusion de la langue latine dans les assises du logos grec, constitue "l'événement proprement dit " de la mutation (Wandlung) de l'essence de la vérité dans l'histoire de l'être. Davantage encore, ce moment inaugural de la mutation de l'essence de la vérité marque "le passage de la "traduction originaire", intra-idiomatique à la traduction au sens courant, la traduction inter-linguistique. La traduction inter-linguistique "ouvre" l'histoire : inaugure une temporalité successive, une temporalité de la mésentente et de la catastrophe " (Escoubas, [1989], p. 117).

Puisqu'Éliane Escoubas nous parle ici de catastrophe, il est opportun d'ouvrir une brève parenthèse (en supplément d'enquête, disons, à son excellente étude) et de considérer les circonstances dans lesquelles Heidegger a dû livrer les fruits de sa méditation à ses étudiants. Nous sommes au semestre d'hiver 1942-1943, en janvier ou en février, alors que l'armée soviétique vient de refermer son étau sur les forces allemandes dans les rues désertes de Stalingrad. L'Allemagne venait de perdre la guerre. Peu de gens le savaient mais Heidegger, lui, le savait. C'était le commencement de la fin. Et le commencement de la fin ne peut manquer de se traduire, doit trouver sa traduction dans l'idiome qui cultive une " pensée du commencement " (anfangliches Denken). I1 s'agit de retracer le point de bifurcation où s'est engagée l'errance fatale (Virerrung), ce dérapage qui a suivi son cours et trouvé son aboutissement inéluctable, entre autres, dans l'assomption de la volonté vers la puissance (Wille zur Macht) chez Nietzsche (Heidegger est extrêmement virulent à son endroit dans ce cours). En fait, comme l'a fort bien démontré Agnes Heller ${ }^{34}$, le cours sur

${ }^{34}$ Agnes Heller, * Parmenides and the Battle of Stalingrad w, in Graduate Faculty Philosophy Journal, New School for Social Research (New York), Vol. 19, No 2-Vol. 20, No $1: \propto$ In Memoriam Reiner Schurmann * (1997) pp. 247-262. 
Parménide est truffé, pour ne pas dire saturé de renvois à connotation politique qui forment en quelque sorte le système nerveux de ce texte et qu'il faut décoder comme tel.

Que ce texte se préoccupe aussi de problèmes de traduction vient tout simplement confirmer ce lien souvent inavoué, parfois inavouable, entre la politique de la traduction et la traduction du politique. Nous pouvons aussi traduire les traductions de Heidegger. Le professeur de Fribourg n'est, à tout prendre, que l'épigone tardif d'une mentalité diffuse dans l'intelligentsia allemande - la Bildung si vous préferez - qui, au moins depuis le XVIII ${ }^{e}$ siècle, affiche une allergie trés marquée à l'endroit de la culture romaine, par contraste avec la pureté de l'aurore grecque qui ne saurait trouver un écho profond qu'en terre du Ponant, dans la contrée de l'Hespérie (Abendland) qui, fort heureusement, est servie par la culture (et la langue) allemande, laquelle nourrit des " affinités électives ", sinon trahit une profonde gémellité avec l'antique civilisation ionienne. Évidemment, pour Heidegger la langue latine est l'idiome de prédilection de la métaphysique. Mais aussi celui du droit (ius), du casus à travers lequel on peut décoder l'aboutissement de la filiation de falsum à fallere en latin, de Falsche à Fall en allemand. Le circuit idiomatique de la " chute " est aussi celui de la iusticia et de la certitudo qui confortent l'espèce métaphysique de la veritas. Mais il y a plus. Le mot falsum, apprend-on par ailleurs, est relié à l'astuce, à la ruse, au subterfuge. Rien d'étonnant là-dedans, nous dit Heidegger, puisque cette contamination vient du mot anglais trick (Trïck en allemand), donc de la langue anglo-saxonne qui, avec les langues de souche romane, est dépositaire du mode de représentation métaphysique : « Zum 'Römischen', écrit Heidegger, gehört auch das 'Romanische' und alles von ihm aus bestimmte neuzeitliche Wesen..." (GA 54, p. 78), en gros, tout ce qu'il y a de moderne, en passant par le « romanique ", ou le " roman " (on pourrait sans doute inclure le genre littéraire), procède du * romain *. L'errance du peuple allemand, sa défection à l'endroit du giron grec, est de s'être plié, conformé à cette sphère d'influence dont l'ultime 
tromperie serait scellée dans l'idéal de la paix, de la pax romana, du pacte qui permet de leurrer les vaincus, de les amener à tomber (d'où le Zu-Fall-Bringen), de les asservir en les élevant, en les "fixant " dans les limites prescrites par la conclusion de l'armistice. Cette façon de " fixer * (abstecken) quelqu'un dans des liens ou des limites vient du latin pango, d'où le mot pax (GA 54, pp. 60-61). Un ticket pour Yalta, où les forces alliées, sous la conduite d'Albion la perfide, conspirèrent contre le peuple dont le destin coïnciderait avec celui de l'Abendland. S'il y a un sous-texte (un pré-texte) à ce texte, c'est cette faille, cette veine noire, cette lésion profonde qui court en filigrane de son interprétation, bref l'inavoué, sinon l'inavouable d'une pensée blessée, humiliée par l'échec de son investissement naïf, et sinistre à plus d'un titre, dans un projet de régénération pédagogique de l'université allemande, lors de la courte période de rectorat de Heidegger. Cette misère emprunte un tour loufoque lorsque Heidegger, à maintes reprises dans ce cours, se lamente sur le fait que la machine à écrire, la dactylographie - un produit de la techné, une excroissance, sinon le fer de lance de la métaphysique - est en train de remplacer l'écriture manuelle, alors que des milliers de soldats allemands sont traqués à Stalingrad et que des millions d'enfants, de femmes et d'hommes juifs sont livrés aux chambres à gaz. Sans doute estce un oubli de l'être.

La question que pose Éliane Escoubas n'en est pas moins pertinente : pourquoi ce privilège accordé à la langue grecque? Ce statut tient certes à son ascendant historique, qui l'habilite comme langue de la pure présence (die vorliegende Sache selbst), par opposition avec un langage référentiel, tout entier voué à la médiation qui fixe la référence d'une intention signifiante, de la Bedeutung. L'opposition majeure qui régit le texte heideggerien est donc celle du nennen et du bedeuten, du " nommer " et du " signifier " : " Le nom est "la chose même" : il ne signifie rien, il est le mode langagier de la présentation - il présente et se présente. Il n'y a pas de traduction inter-linguistique du nom, le nom relève d'une traduction-citation : il est "cité" dans plusieurs 
langues - un nom, comme un idiome, peut traverser plusieurs langues. Ainsi, un nom est un idiome, un idiome est un nom * (Escoubas, [1989], p. 118). Par conséquent, dans la mesure où il y va toujours de la vérité et des formes mêmes de son occultation, il ne saurait y avoir pour Heidegger de bonnes ou de mauvaises traductions, suivant ici l'adage italien traddutore traditore, puisqu'on donnerait alors prise à l'arbitraire, à « la possibilité pour ce qui a eu lieu de n'avoir pas eu lieu ". Le topos de la traduction est plutôt celui de l'impensé, sa mise en cuvre comme réserve de l'histoire de l'être : ce qui est réservé à l'élucidation à laquelle l'être lui-même destine la pensée qui se découvre dans l'horizon " historial " lui octroyant la mesure de son inscription dans le cercle herméneutique où s'opère la a traduction originaire \#, à savoir le saut (Sprung) requis pour s'instaurer dans la langue appropriée au décret de l'être, dans l'élément le plus originel du commencement (ursprünglichere Anfang) qui, cependant, ne devient perceptible que dans la phase la plus avancée d'un déclin dont est synonyme le nom même d'Occident.

Quoiqu'Éliane Escoubas n'en touche pas mot, il apparaît que ce texte où l'on a droit de la part de Heidegger à sa méditation la plus élaborée sur l'essence de la traduction, est habité par une démonique exacerbée, déchirée, qui a trouvé refuge, depuis 1935 au moins, dans l'aura dans laquelle s'enveloppe la Dichtung hölderlinienne, celle qui est pénétrée du climat de la Götterdämmerung, de la * désertion des dieux *. Comment donc retracer le fil de l'intertexte qui censément se tisse entre ce texte et le propos de Benjamin dans Die Aufgabe des Übersetzers?

Nous l'avons déjà noté plus haut, Escoubas considère que ce sont les difficultés et les apories de l'essai rédigé par Benjamin en 1916, "Über Sprache überhaupt und über die Sprache des Menschen " qui auraient entraîné le déplacement sur le terrain de la traduction dans les prolégomènes de 1921. Si l'on s'en tient au synopsis qu'elle propose de la topique de l'essai de 1916, son projet réside dans la détermination de la notion de langue en 
général à partir de la notion d'expression et dans la distinction cardinale entre la " langue des choses" comme élément de la communication et la " langue des hommes " comme celui de la nomination. Ce projet correspond à une double investigation : d'une part, la recherche d'une unité essentielle (la langue) dans la multiplicité de ses modes, la "langue en général " étant alors déterminée comme expression; de l'autre, la tentative pour distinguer, autour de ce centre, des niveaux ou des * séries * de langues correspondant à une distribution hiérarchisée qui obéirait alors à un schéma platonicien, à un chorismos initial qui s'articule autour de la distinction cryptique entre les " essences langagières " et les " essences spirituelles ". Cette lecture, disons-le sans trop y insister car là n'est pas le but de l'exercice, est plutôt problématique. D'abord, rien ne permet d'y lire une détermination de la langue en général à partir de la notion d'expression, celle-ci appartenant du reste à une conception référentielle du langage qui est littéralement taillée en pièces dans cet essai. En fait, la notionclef de l'argumentation transcendantale développée dans cet essai est celle de la communicabilité. Par ailleurs, tel que nous l'avons déjà constaté, la distribution hiérarchisée qui parcourt les divers degrés d'existence dans la vie langagière repose sur un rapport de proportionnalité et son vecteur est celui de la densité, c'est-à-dire qu'elle est d'ordre graduel et non pas sériel. Voyons néanmoins comment s'articule chez Escoubas le passage d'un texte à l'autre, où nous percevons plutôt une radicalisation et un approfondissement des thèses avancées en 1916.

Le Vorwort aux traductions de Baudelaire s'attache exclusivement à la " langue des hommes ", qui est embrassée dans sa condition plurale, post-babélienne : les langues mobilisées par l'homme nous exposent à une multiplicité irréductible. La détermination de cette pluralité n'est plus le fait de séries hiérarchisées, mais répond plutôt à l'idée d'une connexion (Zusammenhang) essentielle qui, à son tour, détermine un concept purement relationnel de l'essence des langues alors perçues à travers le prisme de la traductibilité. Bref, c'est " le rapport qui 
détermine les éléments; l'essence est le rapport lui-même : la traductibilité est l'essence-langue des langues ^ (Escoubas, [1989], p. 121). Autrement dit, on a toujours déjà affaire à l'x entre-deuxlangues ", et cette tension duale se complique d'entrée de jeu d'une asymétrie entre l'intention de l'œuvre à traduire et son éventuelle réception : l'œuvre jouit d'une indemnité absolue, voire d'une totale immunité par rapport à sa réception. Et il en va de même pour sa traduction. La question n'est pas de savoir à qui ou à quoi se destine la traduction, mais d'où provient son désir, son abandon à une tâche qui doit trouver sa motivation non pas dans la possibilité de dire le " même " dans l'" autre ", ou de procéder à une bijection idéale du " même " dans deux sous-ensembles ou champs d'expression différenciés qu'on rendrait compatibles au moyen de la traduction, mais dans le rapport intensif qui fait écho à une mutualité d'intention originelle qui n'existe pas en dehors de ce rapport. D'où l'hypothèse de départ d'Escoubas, selon laquelle l'insuffisance de la détermination de la langue comme expression, dans l'essai de 1916, serait ici relayée par une logique de la connexion. En fait, le propos de Benjamin s'inscrivait déjà, dès 1916, dans une direction opposée de celle qui s'attache à l'extension du domaine du lisible ou du " compréhensible " (verstehbar). Il ne s'agit pas ici de cultiver l'obscur pour l'obscur, mais bien de démarquer l'épreuve de la signifiance d'une conceptualité qui demeure étrangère, insensible à l'étrangeté même des langues. Le pas est donc franchi de façon encore plus décisive dans l'essai de 1921, où Benjamin tente de court-circuiter, par l'approfondissement du Zusammenhang (la connexion, le nexus), du " tenir-ensemble " (zusammen-hängen) d'éléments absolument disparates, toute conception extensionnaliste ou référentielle de la traduction, de sorte que la motivation du " traduire "se résout plutôt en faveur de l'immotivé, à la rigueur dans le voisinage de l'abîme (Abgrund), de l'absence de fondement qui caractérise l'éclosion plurale des langues. L'objet de ce renversement, comme le précise Escoubas, c'est " la "critique" de la notion de communication (Mitteilung) - ou, ce qui revient au même, de la notion d'énonciation (Aussage) et de transmission (Übermittlung) : 
une critique de la raison communicative donc ". (Ibid., pp. 127128).

Le déplacement majeur qui s'opère ici, et c'est là l'hypothèse de lecture d'Escoubas, est que la traduction n'est pas seulement le lieu et l'instrument de cette critique, mais devient dans le texte de Benjamin l'essence même de la langue, de l'être-langue des langues. Pour renforcer cette hypothèse, qui est tout à fait justifiée à notre sens, rappelons que Benjamin renvoie dos à dos comme nuls et non avenus aussi bien une motivation mimétique du vocable qui en ferait une hypostase du réel que le décret arbitraire présidant à l'établissement d'un dispositif sémiotique qui procéderait d'une acculturation contingente. Benjamin s'en tient à une affirmation lapidaire et catégorique qui, nous l'avons déjà suggéré, est le point d'orgue des multiples variations où il tente de cerner ce point de tension fugitif, cette tangente furtive qui voit affleurer dans la disparité des langues le frayage diasporique de la reine Sprache : " La traduction est une forme - Übersetzung ist eine Form ". Pour s'enquérir de la teneur de cette forme, il faut effectuer un retour (zurückgehen) à l'original, c'est-à-dire que la loi (Gesetzt) de cette forme ne réside pas en aval, du côté d'une réception liée à l'impératif de la communication - et de la transmission, mais en amont, comme loi immanente à l'original. Cette loi est celle de la " traductibilité » (Übersetzbarkeit) qui cependant n'est pas l'apanage exclusif de l'original, car elle n'est autre que la connexion, le Zusammenhang de la traduction et de l'original. En fait, il n'y a ici ni amont ni aval, ni envers ni endroit, plutôt un nœud, une espèce d'anneau de Möbius. Cette connexion est natürliche, nous dit Benjamin, c'est une " connexion de vie * (Zusammenhang des Lebens) : la différence des langues n'est pas l'indice d'une séparation, d'un chorismos initial, mais une manière de se " différer ", de se déférer l'une à l'autre. Sans doute y a-t-il lieu de rapprocher ce nœud vivant, dont Benjamin nous disait déjà dans l'essai de 1916 qu'il est un " continuum de métamorphoses " (eine Kontinuum von Verwandlungen), de l'inscription pré- 
originaire de la différance chez Derrida ${ }^{35}$, et ainsi de conjuguer à l'incommunicable la motion disruptrice de l'indécidable, mais il y a aussi chez Benjamin ce " coup de dés " qui jamais n'abolira le hasard, qui est l'instant de la décision et qui ne peut faire l'économie de sa propre déchirure, puisqu'elle n'a d'autre horizon que celui d'une rédemption qui, bien que incessamment différée, pointe dans la disparité des langues vers le " terme messianique de leur histoire ".

Rompant avec la logique de l'extension, cette dynamique se dessine dans l'investissement micrologique de symétries brisées obéissant plutôt à une logique de la contiguitté, laquelle est symbolisée, nous l'avons vu, par le motif kabbalistique du " vase brisé ", de la " brisure des vases "(Shevirath ha-kelim) qui correspond, si l'on s'en fie à la dramaturgie du mythe lourianique, à l'éclatement et à la dissémination de l'énergie divine, à l'épanchement d'une * crise » dans le retranchement le plus intime de l'ipséité divine et dont la cassure donnera le " branle " à l'histoire, à l'exil de la créature. Cette logique de la contiguîté est aussi typifiée par le symbolon grec lui-même, c'est-à-dire l'agencement discret de parties absolument dissemblables permettant de renouer des alliances. La dissymétrie des formes de la vie langagière garantit la possibilité même d'un rapprochement, de ce contact fugitif qui correspond sans doute à l'essence même de la manifestation du vivant qui est tributaire de ruptures d'équilibre qui seules permettent l'émergence du nouveau, de l'inédit. Cette dissymétrie confirme l'unicité du singulier en même temps que l'inachèvement de la forme scellée dans les constructions finies de la langue. La trace de cette harmonie lointaine, à peine perceptible et vouée à l'oubli parce que inoubliable, ne peut qu'affleurer dans la traduction qui actualise, sur un mode purement intensif et anticipatoire, l'" affinité "

${ }^{35}$ Voir à ce sujet Bettine Menke, Sprachfiguren. Name - Allegorie - Bild nach Walter Benjamin (München, W. Fink Verlag, 1991). 
(Verwandtschaft) que les langues nourrissent à leur insu, dans la singularité même des formes d'expression auxquelles elles donnent lieu et qui creusent la frontière intérieure de leur horizon mis en abyme dans l'épreuve de l'étranger. La pluralité des langues signifie aussi que la contiguiité de la forme et du contenu, de la teneur (Gehalt) et de la langue, admet divers degrés d'adhérence eu égard à leur fusion spécifique dans la facture de l'original. C'est pourquoi Benjamin recourt de façon expresse à la notion de " complémentarité * (Ergänzung) pour caractériser cette affinité purement intensive, donc non extensive, c'est-à-dire indépendante du contenu visé ou véhiculé par la forme de l'expression, répudiant du même coup la thèse de la substitutivité des langues et le régime de l'équivalence comme tuteur transcendantal de la traduction. Autrement dit, une langue n'est jamais seule, " une langue n'est "langue" que d'être l'autre d'une autre langue ". (Escoubas, [1989], p. 131)

Cette " connexion de vie " entre une æuvre et sa traduction, en cela même qu'elle touche au devenir des langues, situe la micrologique de la contiguité et de l'affinité dans l'envergure d'une " descente infinie " des noms disséminés dans la diaspora des langues et dont l'errance est nouée au vecteur temporel de l'histoire, au sens où l'entendait plus haut Giorgio Agamben. Escoubas saisit dans le jeu subtil des préfixes über- et fortappliqués à cette connexion de vie, soit la Überleben, la a survie " de l'œuvre, et la Fortleben, la continuation inespérée de sa vie, cet écart intime qui lie la transcendance de l'œuvre, son autonomie absolue en regard de sa réception et de sa traduction, à sa loi immanente, celle de la traductibilité qui déjà l'extrade hors de son lieu de naissance et l'inscrit dans un ordre de croissance qui excède son aire de rayonnement. Ce mouvement d'exode, cette tension exodique qui nourrit secrètement la poétique de l'œuvre, est la condition même de la relation d'inconnu qui l'ouvre de l'intérieur et dont le point de fuite est encodé dans le rapport à l'étranger qui déjà délite la structure de l'œuvre, la désature et marque ainsi le plan d'effraction de l'histoire dans l'unicité de sa forme, sa 
cicatrice si l'on ose dire. Comme le note Escoubas, dans le texte de Benjamin la notion de Fortleben est ausitôt relayée par celle d'Entfaltung, soit la notion d'un développement purement intensif qui accuse l'effet de rupture, la discontinuité liée à l'œeuvre du temps, comme facteur de croissance et de métamorphose où la traduction se révèle comme " opérateur d'historicité " (Ibid, $\mathrm{p}$. 133).

Sur ce point, nous ne pouvons que renvoyer aux fines analyses de Jeanne-Marie Gagnebin qui propose à notre sens l'une des lectures les plus justes et les plus nuancées de la conception de l'histoire chez Benjamin ${ }^{36}$. Le temps historique, observe-t-elle, est conçu chez Benjamin en termes d'intensité et non de succession chronologique. Ce qui signifie que le concept d'origine (Ursprung), loin de référer à quelque status quo ante déterminé par une conception génétique de la temporalité, désigne plutôt le " saut " (Sprung) qui permet de " faire sauter (heraus-sprengen) le passé figé hors du continu historique ", et ce de façon à * arrêter le temps pour permettre au passé oublié et refoulé de surgir à nouveau (ent-springen, même radical qu'Ursprung), d'être ainsi repris et sauvé dans 1'actuel n (Histoire..., p. 21). La syncope qui brise l'enchaînement infernal des événements est pure fulguration, contraction de la Jetzt-Zeit, de l'intensité de l'instant dans l'objet, inscrivant une césure disruptrice et rédemptrice dans la trame anonyme de l'histoire, soit cette faille ténue, cette anfractuosité où s'illumine, l'espace d'un instant, l'amplitude absolue entre la préet la post-histoire. L'histoire et la temporalité, comme le précise Gagnebin, loin d'être niées par cette contraction paroxystique, vertigineuse, du rythme temporel, se trouvent pour ainsi dire

36 Jeanne-Marie Gagnebin, Histoire et narration chez Walter Benjamin (Paris, L'Harmattan, 1994); nous nous référons principalement ici au chap. 1 ; * Origine, original, traduction ", pp. 17-51; voir aussi * Histoire, mémoire et oubli chez Walter Benjamin * (Revue de Métaphysique et de Morale, n 3/1994), pp. 365389; Zur Geschichtsphilosophie Walter Benjamins. Die Unabgeschlossenheit des Sinnes (Erlangen, Verlag Palm \& Enke, 1978). 
" concentrées dans l'objet : relation intensive de l'objet au temps, du temps dans l'objet, et non extensive de l'objet dans le temps, placé comme par accident dans un déroulement historique hétérogène à sa constitution n (Ibid.).

Nous reportant aux trois modèles sous-jacents à la Préface de l'étude sur le Trauerspiel, à savoir la théorie de l'Urphänomen chez Gothe, la théologie juive de l'exil et de la rédemption et la doctrine platonicienne des Idées, Gagnebin rappelle que si le concept d'origine ne " tombe " pas lui-même sous le concept, s'il y résiste d'une résistance qui évolue à fonds perdu, dans la fragilité de sa dispersion, toujours au bord du naufrage, c'est que l'origine est à la fois " indice de la totalité et marque éclatante de son manque; dans ce sens précis, elle renvoie bien à une temporalité resplendissante, celle de la promesse et du possible qui surgissent dans l'histoire " (Ibid., p. 25). De la même façon, l'évocation récurrente chez Benjamin d'une restitutio in integrum, ou encore de la notion d'apokatastasis empruntée à la doctrine sotériologique d'Origène, doit aussi être conçue à partir de l'inachèvement d'une totalité qui ne saurait se refermer sur elle-même : l'idée d'une restauration est résolument orientée vers le futur, comme anticipation d'un royaume qui ne peut être entrevu que dans la dispersion rhapsodique des éléments qui, si l'on peut dire, " espèrent n le nom, $y$ aspirent et le sollicitent à l'instar des étoiles qui, perdues dans l'immensité muette de la nuit cosmique, n'échappent à leur fossilisation inéluctable qu'en épousant pour un temps le tracé d'une constellation, qu'en recevant son nom où se cristallise la co-appartenance de l'éternel et de l'éphémère. C'est bien parce que la " langue des noms " est à jamais perdue que la dérive des langues $\mathrm{y}$ aspire secrètement, non point dans un élan de nostalgie désireux de renouer avec une condition idyllique, mais dans la brisure de l'écorce du fruit qui, venu à maturité, révèle une semence grosse d'une promesse qui ne tient qu'à la saisie de l'instant, du kairos où l'émergence du nouveau libère les forces enfouies sous les ruines de Babel. Autrement dit, 
la dynamique de l'origine ne s'épuise pas dans la restauration d'un stade premier, que celui-ci ait véritablement existé ou qu'il soit une projection mythique vers le passé; parce qu'il est aussi inachèvement et ouverture sur l'histoire, surgissement historique privilégié, l'Ursprung n'est pas simplement restauration de l'identique oublié mais aussi et inséparablement émergence du différent. Cette structure paradoxale est celle de l'instant décisif, du Kairos. (Ibid., p. 31)

Cette conception kairologique de l'idée d'origine signifie qu'y est tendue l'amorce d'une transmutation de la facies hippocratica, douloureuse, de l'histoire comme catastrophe d'où il faut rescaper la face symbolique cachée, l'harmonie originelle du nom enfoui dans la disparité des langues; bref, c'est " dans la différence entre les langues, dans cet intervalle douloureux que le traducteur prétend, à première vue, combler, mais qu'en vérité il révèle dans sa profondeur, que peut s'exposer leur vérité * (Ibid., p. 33). Ainsi, l'exigence de la « réparation " (Tikkun) dans le mythe lourianique, qui doit prendre sur soi le sens et la portée de l'exil de la créature à la suite de la Shevira, de l'éclatement des vases où étaient contenus les germes de l'énergie divine, ne saurait les restaurer, les restituer à leur condition initiale : il est possible de ne saisir qu'une étincelle de la lumière primordiale, et c'est cette * faible force messianique " qui seule permet de réactualiser, sans jamais la retrouver, l'harmonie profonde de la " langue des noms". L'univers s'éloigne à jamais de l'ordre premier qui présida à son éclosion, et dans la trace de ce retrait du Nom divin hors de la sphère du dicible se dessine toute l'épaisseur de l'histoire, qui n'est autre que la douleur de l'exil pressentie dans la différence des langues rivées à cet abîme inscrutable que la traduction ne peut combler, mais doit expérimenter pour ressourcer le secret dessein qui les traverse sans qu'elles puissent en assumer la maîtrise, en arrêter la forme définitive. Dans un fragment vraisemblablement rédigé en 1920-1921 et intitulé "Sprache und Logik ", Benjamin rappelle que la multiplicité des langues se présente comme une pluralité d'essences. Cet état de choses, poursuit-il, n'est pas la 
conséquence d'une dégradation, d'une dégénérescence ayant affecté une langue première, unique, qui eût été en possession de locuteurs issus on ne sait d'où, mais plutôt l'indice composite de la perte progressive de la puissance d'évocation de l'harmonie régissant originellement le rapport entre des langues qui sont multiples par essence, cette essence n'étant autre que celle de 1'histoire. La doctrine des mystiques, écrit-il, " ne peut aller dans le sens de sa [la vraie langue, die wahre Sprache] dissolution dans une multiplicité, mais doit bien davantage référer à une perte progressive de puissance de la force de maîtrise intégrale, celle-là même qu'on voudra attribuer, dans le sens des mystiques, à un mode de signification d'ordre langagier doué d'une unité d'essence révélée (einer offenbarten Wesenseinheit sprachlicher Art der Bedeutung), de sorte que celle-ci [la vraie langue] ne serait pas tant apparue comme la langue originelle réellement parlée, mais beaucoup plus comme l'harmonie qui à l'origine s'est rendue perceptible à partir de toutes les langues parlées, une harmonie chargée d'une force langagière incommensurablement plus grande que celle dont est dotée n'importe quelle langue vernaculaire. $x^{37}$

Le degré de complexité auquel est confrontée la traduction dans l'abord d'une poétique déjà surcodée sur le plan des tropes, de la syntaxe, de la prosodie, sans compter l'horizon de référence immanent à la facture d'une œuvre, l'instruit sur-le-champ comme "opérateur d'historité ", suivant l'expression d'Escoubas. I1 ne s'agit pas de négocier une solution de continuité, de combler un déficit sinon de conjurer l'entropie qui présumément menacerait l'intégrité du sens, mais d'accentuer, de marquer, de révéler dans l'unicité de son occurrence, et l'indélébilité de sa trace, ce que nous désignons volontiers comme la cicatrice du temps, la blessure de l'histoire. La poétique de l'œuvre, la Dichtung, est le nœud d'un développement ininterrompu, une matrice ouverte à la mutation

${ }^{37}$ W. Benjamin, "Sprache und Logik " (II), in G.S. VI (éd. par Tiedemann et Schweppenhauser), pp. 24-25. 
(Wandlung), la croissance (Wachstum) et la maturation (Nachreife). Comme le note Escoubas, l'entrelacs qui lie la traduction à ce noud vivant est tissé d'un double paradoxe, en vertu duquel, d'une part, la traduction anticipe (vorgreifen) l'avenir de l'œuvre, l'indique à l'avance (andeuten), s'inscrivant alors dans un rapport d'anticipation plutôt que sous la rubrique de la conservation ou de la thésaurisation, alors que, d'un autre côté, elle est dépassée par cette croissance à laquelle elle participe, celle de l'œuvre et des langues qui s'y traduisent en la traduisant, puisqu'il appartient à l'original d'être incessamment retraduit or que toute traduction singulière a pour destin et propriété intrinsèque d'être frappée de caducité, donc de " périr et de disparaître " (ein und untergehen).

Cette vulnérabilité de la traduction n'est pas le fait d'une servitude acquise à l'ascendant tutélaire d'un signifié transcendantal. La visée du traducteur n'est autre que celle de l'historien, mais n'opère qu'à l'affût d'une tangence furtive, absolument passagère, qui vise l'affinité supra-historique des langues. Cette supra-historicité, dans la mesure où elle vise le terme messianique de leur histoire, $s$ 'appuie davantage sur leur incomplétude, s'y ressource même, plutôt qu'elle n'a dessein d'y pallier, à la façon, par exemple, de l'Aufhebung hégélienne, qui ne conserve le moment singulier que pour le supprimer et l'intégrer au devenir du concept. La singularité idiomatique de l'élément visé par la traduction est plutôt la marque d'un éloignement (Entfernung) qu'il faut serrer au plus près pour y saisir les harmoniques du " pur langage ". À notre sens, nous pouvons rapprocher ce souci micrologique apporté à l'indice historiocritique de la forme langagière du réquisit formulé par Benjamin dans ses Thèses sur la philosophie de l'histoire, selon lequel * le passé apporte avec lui un index temporel qui le renvoie à la délivrance. Il existe une entente tacite entre les générations passées et la nôtre. Sur Terre nous avons été attendus. À nous, comme à chaque génération précédente, fut accordée une faible force messianique sur laquelle le passé fait valoir une prétention " 
(Poésie et révolution, p. 278).

Aussi Éliane Escoubas a-t-elle absolument raison de lire en filigrane de l'essai sur la traduction, notamment en ce qui touche à l'intégration des langues humaines, "la constitution chez Benjamin du concept cosmo-politique d'humanité " (Escoubas, [1989], p. 135). Cette fonction cosmo-politique est précisément ce que Benjamin nomme " die Lehre ", la " doctrine ", et, dans ce cas, comme le suggère Escoubas, la tangente messianique ou encore l'éthique rédemptrice qui sous-tend sa théorie de la traduction viserait, mutatis mutandis nous le précisons, ce qui chez Heidegger correspond à l'impensé de l'histoire, une histoire qui pour ce dernier ne saurait cependant être autre que celle de l'être. Qui plus est, le spectre sémantique qui, dans l'essai de Benjamin, ressortit à la logique de la connexion, du Zusammenhang, et qui unit contiguité, affinité et complémentarité, correspondrait à l'articulation duplice de l'idiomatique et de l'ekstatique chez Heidegger. Mais il faut aussi composer avec " cette schize, (...) cette "interminable cassure" (unaufhörliche Zwiespalt), qui déchire la traduction " (Ibid., p. 136), celle qui renvoie dos à dos fidélité et liberté. On ne peut transiger avec cette déchirure : la Wörtlichkeit, la littéralité ne saurait être bradée au profit de la conservation du sens. Si le mot est " l'élément originaire du traducteur " (das Urelement des Übersetzers), alors, écrit Benjamin, "la restitution du sens doit cesser de faire la loi *. I1 y a un reste irréductible, un élément intangible, un " non-communicable " (eine NichtMitteilbares) qui n'est autre que la forme de l'original. Celle-ci renvoie à la " langue des noms ", ces noms dont chacun constitue, suivant l'analyse d'Escoubas, un idiome. Dès lors, l'impératif de la Umdichtung, de la transmutation poétique qui doit a racheter dans sa propre langue cette pure langue captive dans la langue étrangère " correspondrait à une traduction intra-idiomatique qui aurait déjà fait son deuil de l'atomisme des significations et du fétichisme du sens propre. Autrement dit, le souci de la littéralité chez Benjamin, la justification de son réquisit, n'a absolument rien à voir avec un littéralisme creux et plat. Ainsi l'Urbild de la 
traduction, son archétype, n'est-il pas un paradigme anhistorique qui réduirait au néant, sinon aux ressorts de l'artifice et de l'expédient, toute tentative pour s'affranchir de son ascendant, mais ce point de tension de l'energeia (toujours au sens humboldtien) par laquelle s'élaborent simultanément, et convergent donc toujours de quelque façon, le devenir-cuvre d'une langue et le devenir-langue d'une ceuvre.

L'indivision de la langue, dont le postulat est d'un commun partage chez Heidegger et Benjamin, signifie qu'elle est elle-même en constante métamorphose. La figure complexe de cette dynamique correspond au descendendo infini de l'histoire où la traduction est toujours déjà impliquée, sur un mode intensif, originaire et anticipatoire, comme « faible force messianique. $\mathrm{Si}$ elle est partie prenante du concept cosmo-politique d'humanité, c'est qu'on ne saurait faire l'économie, donc réduire à l'uniformité d'un chiffre ou d'un code, la diaspora des langues où nous faisons l'apprentissage, certes toujours problématique, d'un commun partage de l'œkoumène. Sans doute y a-t-il lieu de pressentir dans cette tension nodale de l'entre-deux-langues * l'articulation sans cesse déchirante et sans cesse renouante de l'idiomatique et de l'ekstatique "(Ibid., p. 142), telle qu'elle se découvre précisément dans la méditation de Heidegger, mais l'enjeu qu'elle revêt chez Benjamin est de loin beaucoup plus radical, fragile et désespéré. On ne saurait réinventer, et même simplement inventer une rencontre qui n'a jamais eu lieu, mais l'abîme qui sépare Benjamin de Heidegger est de ceux que les mots ne savent combler. Aussi allons-nous clore cet examen, toujours préliminaire, des rites de l'interprétation autour de l'essai sur la traduction en citant ce passage d'un fragment rédigé par Benjamin en 1921, et lui aussi resté inédit de son vivant, * le Rôle du temps dans le monde moral ", ici dans la traduction de Patricia Konigsberger :

Comme l'ouragan purificateur annonce la tempête, la colère de Dieu inspire la tempête du pardon qui traverse l'histoire pour venir balayer tout ce qui dans les foudres divines du temps devrait être 
consommé pour toujours. Ce qui est dit dans cette image doit se laisser traduire clairement en concepts; dans l'économie du monde moral à l'intérieur duquel s'effaceront les traces du crime, le rôle du temps, au-delà du souvenir et de l'oubli, sera énigmatiquement d'amener, par sa durée, le pardon mais jamais la réconciliation. ${ }^{38}$

\title{
Université de Montréal
}

RÉSUMÉ : La déshérence du clandestin : les rites de l'interprétation autour de l'essai sur la traduction de Walter Benjamin - À partir d'un corpus restreint, bien délimité, d'analyses critiques consacrées à l'essai sur la traduction de Benjamin, cette étude dégage une diversité de points de vue qui, sous leur contradiction apparente, en appellent de la part du lecteur à un processus de traduction continue qui est pour ainsi dire encodée, de façon intrinsèque, dans la poétique même de Benjamin. Loin de se vouloir un cas d'espèce enveloppé dans une aura ésotérique, cette façon de résister à toute forme d'explaining away, indique plutôt que toute théorie de la traduction doit ellemême se * traduire * dans l'élément où elle tente de s'expliquer.

\begin{abstract}
The Clandestine without Heirs : Rites of Interpretation Practiced on Walter Benjamin's Essay on Translation - Based on a well-defined corpus of critical appraisals of Benjamin's essay on translation, this article examines the various interpretations the work has generated. Their apparent contradictions require of the reader a sustained process of translation, a process which is, so to speak, already encoded in Benjamin's poetics. Far from being a case of esoteric musing, this critical resistance to any kind of " explaining away * indicates
\end{abstract}

${ }^{38}$ W. Benjamin, - le Róle du temps dans le monde moral " (Die Bedeutung der Zeit in der moralischen Welt), manuscrit 798 du Nachlass de Walter Benjamin, trad. par Patricia Konigsberger (Le Nouveau Commerce, $\mathrm{n}^{\circ} 49$, printemps 1981), p. 53. 
rather that any theory of translation must $\alpha$ translate $*$ itself in the element where it attempts to provide an explanation.

Laurent Lamy: Département de linguistique et de traduction, Université de Montréal, C.P. 6128, Succursale Centre-Ville, Montréal (Québec) H3C 3J7. 